\title{
Quantum Mesoscopic Scattering: Disordered Systems and Dyson Circular Ensembles
}

\author{
Rodolfo A. Jalabert ${ }^{(1,2)}$ and Jean-Louis Pichard ${ }^{(1)}$ \\ (1) CEA, Service de Physique de l'Etat Condensé, Centre d'Études de Saclay, \\ 91191 Gif-sur-Yvette Cedex, France \\ ${ }^{(2)}$ Division de Physique Théorique* ${ }^{*}$ Institut de Physique Nucléaire, 91406 Orsay Cedex, France
}

(7 September 1994)

\begin{abstract}
We consider elastic reflection and transmission of electrons by a disordered system characterized by a $2 N \times 2 N$ scattering matrix $S$. Expressing $S$ in terms of the $N$ radial parameters and of the four $N \times N$ unitary matrices used for the standard transfer matrix parametrization, we calculate their probability distributions for the circular orthogonal (COE) and unitary (CUE) Dyson ensembles. In this parametrization, we explicitely compare the COECUE distributions with those suitable for quasi-1d conductors and insulators. Then, returning to the usual eigenvalue-eigenvector parametrization of $S$, we study the distributions of the scattering phase shifts. For a quasi-1d metallic system, microscopic simulations show that the phase shift density and correlation functions are close to those of the circular ensembles. When quasi-1d longitudinal localization breaks $S$ into two uncorrelated reflection matrices, the phase shift form factor $b(k)$ exhibits a crossover from a behavior characteristic of two uncoupled COE-CUE (small $k$ ) to a single COE-CUE behavior (large $k$ ). Outside quasi-one dimension, we find that the phase shift density is no longer uniform and $S$ remains nonzero after disorder averaging. We use perturbation theory to calculate the deviations to the isotropic Dyson distributions. When the electron dynamics is no longer zero dimensional in the transverse directions, small- $k$ corrections to the COE-CUE behavior of $b(k)$ appear, which are reminiscent of the dimensionality dependent non universal regime of energy level statistics. Using a known relation between the scattering phase shifts and the system energy levels, we analyse those corrections to the universal random matrix behavior of $S$ which result from $d$-dimensional diffusion on short time scales.
\end{abstract}

PACS:05.60, 72.10B, 72.15R, 72.20M

Typeset using REVTEX

*Unité de Recherche des Universités Paris XI et Paris VI associée au CNRS 


\section{INTRODUCTION}

The discovery of universal conductance fluctuations [1] 3] (UCF characterizing the sensitivity of the conductance of small metallic samples to a change of the Fermi energy, magnetic field or impurity configuration) have generated a sustained interest in quantum mesoscopic physics since the mid eighties. Mesoscopic systems have a size of the order of the electron phase-coherence length $L_{\phi}$, i.e. a scale intermediate between single atoms (microscopic) and bulk solids (macroscopic). First, it is in terms of quantum interference effects between different multiple scattering paths that UCF has been understood [2, 3]. The universality of the phenomenon (reproducible fluctuations of order $e^{2} / h$, independently of the mean conductance) quickly lead physicists [4,5] to understand UCF as a signature of a more general universality resulting from the eigenvalue correlations of random matrices.

The standard random matrix ensembles were introduced in the context of Nuclear Physics [6] 9] and later found to also describe the statistical properties of quantum systems whose classical analogs are chaotic [10,11]. Contrary to a microscopic approach where the system hamiltonian $H$, scattering matrix $S$ or transfer matrix $M$ result from a more or less arbitrary distribution of the substrate potential, random matrix theory (RMT) assumes for $H, S$ or $M$ statistical ensembles resulting from an hypothesis of maximum randomness, given the system symmetries (time reversal symmetry, spin rotation symmetry, current conservation) plus a few additionnal constraints.

A direct relationship between conductance fluctuations and RMT comes from the Thouless expression of the conductance

$$
G=\frac{e^{2}}{\hbar} N\left(E_{c}\right)
$$

given by the number of one-electron flevels $N\left(E_{c}\right)$ which lie within an energy band of width $E_{c}=D \hbar / L^{2}$ centered around the Fermi energy $E_{f}$. The Thouless energy $E_{c}$ is the inverse characteristic time for an electron to diffuse through the sample (of size $L$ ). The electron diffusion coefficient is $D=v_{f} l / d$, where $v_{f}$ is the Fermi velocity, $l$ the elastic mean free path, and $d$ the spatial dimension of the sample. Fluctuations in the number of levels within $E_{c}$ are therefore related to conductance fluctuations. The analysis of fluctuations in the spectrum of non interacting electrons in metallic particles was initiated by Gorkov and Eliashberg [12]. The relevance of RMT was proved by Efetov [13 and complemented by Altshuler and Shklovskiı [⿴囗 때 who noticed non RMT behavior for energy separations larger than $E_{c}$. Using a diagramatic perturbation theory for the density-density correlation function in the weak disorder limit $k l \gg 1$ ( $k$ is the Fermi wave vector), they showed that the correlation between energy levels is correctly described by the universal RMT laws for energy scales smaller than $E_{c}$, but are weaker (and dimensionality dependent) beyond $E_{c}$. ${ }^{2}$

\footnotetext{
${ }^{1}$ Throughout this work we will treat spinless electrons and therefore we will not write spindegeneracy factors.

${ }^{2}$ This limit of the universal RMT laws is specifically derived ignoring electron-electron interaction.
} 
Pionieered by Imry [0], an alternative approach relating UCF and random matrix theories is based on the Landauer formula, which gives the conductance in terms of the total transmission coefficient of the sample (at the Fermi energy), considered as a single, complex elastic scatterer. For a two-probe measurement where the disordered sample is attached between two perfect reservoirs with an infinitesimal difference in their electrochemical potentials, the conductance (measured in units of $e^{2} / h$ ) is

$$
g=\operatorname{Tr}\left[t t^{\dagger}\right] .
$$

The transmission matrix $t$ can be expressed in terms of the transfer matrix $M$, for which a standard random matrix theory ("global approach") has been developed [14,15]. A set of $N$ real positive parameters describing the radial part of the $2 N \times 2 N$ transfer matrix $M$ (precise definitions are given in the next section) are the relevant "eigenvalues" in this approach, and their probability distribution is

$$
\begin{gathered}
P\left(\left\{\lambda_{a}\right\}\right)=\exp \left(-\beta \mathcal{H}\left(\left\{\lambda_{a}\right\}\right)\right), \\
\mathcal{H}\left(\left\{\lambda_{a}\right\}\right)=-\sum_{a<b}^{N} \log \left|\lambda_{a}-\lambda_{b}\right|+\sum_{a=1}^{N} V\left(\lambda_{a}\right) .
\end{gathered}
$$

This is the usual RMT Coulomb gas analogy with logarithmic pairwise interaction, a system dependent confining potential $V(\lambda)$, and an inverse temperature $\beta=1,2,4$ depending on the system symmetries. This distribution corresponds to the most random statistical ensemble for $M$ given the average density $\rho(\lambda)$ of radial parameters, which controls the average conductance $\langle g\rangle$. In this maximum entropy ensemble, $V(\lambda)$ and $\rho(\lambda)$ are related by an integral relation in the large $N$-limit [16]:

$$
V(\lambda)+C=\int_{0}^{\infty} \mathrm{d} \lambda^{\prime} \rho\left(\lambda^{\prime}\right) \log \left|\lambda-\lambda^{\prime}\right|+\frac{\beta-2}{2 \beta} \log \rho(\lambda) .
$$

$C$ is a constant, and to leading order in $N$ this mean field equation expresses the equilibrium of a charge at $\lambda$ resulting from its interaction with the remaining charges and the confining potential.

In this work we examine the statistical properties of another matrix related to quantum transport in disordered systems: the scattering matrix $S$.

First, the relation between $M$ and $S$ is straightforward, since $M$ can be expressed in terms of the reflection and transmission submatrices which define $S$. This allowes us to show that the $\lambda$-statistics characterizing the Dyson ensembles coincides with a "global approach" for $M$, given a particular confining potential $V(\lambda)$. Using this equivalence, the detailed proof of it being given in this work, the quantum transport properties associated with the circular ensembles have been obtained [17], including the weak-localization effects and the quantum fluctuations of various linear statistics of the $\lambda$-parameters (conductance, shot-noise power, conductance of a normal-superconducting microbridge, etc). This equivalence has been also derived by Baranger and Mello [18], and has been confirmed in their numerical simulations of chaotic billiards. 
Second, since $S$ is unitary, its $2 N$ eigenvalues $\left\{\exp \left(i \theta_{m}\right)\right\}$ are just given by the $2 N$ phase shifts $\left\{\theta_{m}\right\}$. In the Dyson circular ensembles, where all matrices $S$ with a given symmetry are equally probable, the phase shift statistics follow the same universal level correlations than in the gaussian ensembles of corresponding symmetry. The applicability of Dyson circular ensembles for chaotic scattering has been established by the pioneering work of Blümel and Smilansky [19], as far as the two-level form factor for the phase shifts is concerned. Further evidence comes from numerical studies of $2 \times 2$ matrices describing the scattering through chaotic cavities [20].

But a ballistic chaotic cavity, with a conductance of order $N / 2$ and where the electronic motion is essentially zero-dimensional after a (short) time of flight, differs from a disordered conductor or insulator. The introduction of bulk disorder in the dot reduces the conductance to smaller values of order $\mathrm{Nl} / L(L>>l$, metallic regime) and enhances the time interval in which the electron motion depends on system dimensionality. The diffusive motion of the carriers after a (short) characteristic time $\tau_{e}$ limits (rather than improves) the validity of the standard RMT distributions. The subject of this study consists precisely in understanding this apparent paradox: the fact that the introduction in a cavity of bulk disorder drives the statistics of $S$ away from the circular ensembles. For this purpose, we extensively study disordered systems where non-interacting electrons are elastically scatterered by microscopic impurities contained in a rectangular dot of various aspect ratios. We derive useful relations between different parametrizations of $H, M$ and $S$, involving the energy levels $\left\{E_{i}\right\}$, the radial parameters $\left\{\lambda_{a}\right\}$ and the phase shifts $\left\{\theta_{m}\right\}$.

The paper is organized as follows. After presenting the basic definitions of our model and the relationship between $S$ and $M$, we derive (Sec. III) the metric of $S$ in the polar decomposition, and therefore the probability distribution of the radial parameters assuming a Dyson distribution for the scattering matrix in the time-symmetric case (the case without time-reversal symmetry is worked out in Appendix D). In Sec. $\mathbb{D V}$ we use this parametrization to discuss the differences and similarities between the circular ensemble distributions and those suitable for quasi-one dimensional disordered systems. In Sec. $\nabla$ we start the statistical analysis of the phase shifts of $S$ for long conductors with weak disorder. In Sec. V1] we study the scattering phase shifts in quasi- $1 d$ insulators and show that their correlations are well described by those of two uncoupled circular ensembles. In Sec. VII we calculate by diagrammatic perturbation theory the mean values of the $S$-matrix, which are needed to understand the phase shift anisotropy found outside the quasi- $1 d$ limit. In Sec. VIII we present numerical results for geometries other than quasi-one dimensional strips (i.e. squares and thin slabs) and show that after the spectrum is unfolded there is a rough agreement with the circular ensembles, though noticable discrepancies are visible in the number variance. These deviations are analyzed in Sec. [X], using the known deviations of metallic spectra from the R.M.T. behaviors and exploiting a relationship between scattering phase shifts and the energy levels (Appendix B). Appendix $\mathrm{A}$ gives a summary of the methods and results of the numerical simulations, and in Appendix $\mathbf{Q}$, we use a semiclassical approach to complement our understanding of the results, including the meaning of the Wigner time. 


\section{SCATTERING, TRANSFER, TRANSPORT AND $\lambda$-PARAMETERS}

Using only the system symmetries (current conservation, time reversal symmetry, spin rotation symmetry) one can show that both $S$ and $M$ can be expressed in terms of the $N$ radial parameters $\left\{\lambda_{a}\right\}$ of $M$ and 4 (2 in the presence of time reversal symmetry) $N \times N$ auxiliary unitary matrices. In this parametrization, we shall show the similarities and the differences between the distributions implied by the circular ensembles and those describing in the weak scattering limit long quasi-1d disordered conductors and insulators. To make concrete our explanations, we introduce in what follows the essential elements of the particular microscopic model on which we test the validity of Dyson circular ensembles.

We consider an infinite strip composed of two semi-infinite perfectly conducting leads of width $L_{y}$ connected by a disordered part of same width and of longitudinal length $L_{x}$ (Fig. 1). Assuming non-interacting electrons and hard-wall boundary conditions for the transverse part of the wavefunction, the scattering states in the leads at the Fermi energy $E_{f}=\hbar^{2} k^{2} / 2 m$ satisfy the condition $k^{2}=\left(n \pi / L_{y}\right)^{2}+k_{n}^{2}$, where $k$ is the Fermi wavevector, $n \pi / L_{y}$ the quantized transverse wavevector and $k_{n}$ the longitudinal momentum. The various transverse momenta labeled by the index $n(n=1, \ldots, N)$ which satisfy this relationship with $k_{n}^{2}>0$ define the $N$ propagating channels of the leads. Since each channel can carry two waves travelling in opposite directions, asymptotically far from the disordered region the wave function can be specified by a $2 N$-component vector on the two sides of the disordered part. In each lead the first $N$ components are the amplitudes of the waves propagating to the right and the remaining $N$ components are the amplitudes of the waves travelling to the left.

$$
\begin{gathered}
\Psi_{I}(x, y)=\sum_{n=1}^{N} \frac{1}{k_{n}^{1 / 2}}\left(A_{n} e^{i k_{n} x}+B_{n} e^{-i k_{n} x}\right) \phi_{n}(y) \\
\Psi_{I I}(x, y)=\sum_{n=1}^{N} \frac{1}{k_{n}^{1 / 2}}\left(C_{n} e^{i k_{n}\left(x-L_{x}\right)}+D_{n} e^{-i k_{n}\left(x-L_{x}\right)}\right) \phi_{n}(y) .
\end{gathered}
$$

The transverse wavefunctions are $\phi_{n}(y)=\sqrt{2 / L_{y}} \sin \left(\pi n y / L_{y}\right)$. The normalization is chosen in order to have a unit incoming flux in each channel. The scattering matrix $S$ relates the incoming flux to the outgoing flux

$$
\left(\begin{array}{l}
B \\
C
\end{array}\right)=S\left(\begin{array}{l}
A \\
D
\end{array}\right) .
$$

$S$ is a $2 N \times 2 N$ matrix of the form

$$
S=\left(\begin{array}{ll}
r & t^{\prime} \\
t & r^{\prime}
\end{array}\right) .
$$

The reflection (transmission) matrix $r(t)$ is an $N \times N$ matrix whose elements $r_{b a}\left(t_{b a}\right)$ denote the refected (transmitted) amplitude in channel $b$ when there is a unit flux incident from the left in channel $a$. The amplitudes $r^{\prime}$ and $t^{\prime}$ have similar meannings, except that the incident 
flux comes from the right. [5 Current conservation implies that $S$ is unitary. Note that, with the convention we have taken, $S$ for a perfect (non-disordered) sample at zero magnetic field is not the identity matrix but is characterized by transmission submatrices which contain pure phases $t_{b a}=t_{b a}^{\prime *}=\delta_{a b} \exp \left(i k_{b} L_{x}\right)$.

The $2 N \times 2 N$ transfer matrix relates the flux amplitudes on the left-hand side of the disorder part with those on the right:

$$
\left(\begin{array}{l}
C \\
D
\end{array}\right)=M\left(\begin{array}{l}
A \\
B
\end{array}\right) .
$$

Just as for $S$, we can write $M$ in terms of four $N \times N$ blocks

$$
M=\left(\begin{array}{ll}
m_{1} & m_{2} \\
m_{3} & m_{4}
\end{array}\right) .
$$

The reflection and transmission matrices of $S$ can be expressed in terms of the block matrices $m_{i}$ of $M$. Introducing the polar representation [21,22] of $M$, we have:

$$
\begin{gathered}
r=-m_{4}^{-1} m_{3}=-u^{(3)} \mathcal{R} u^{(1)} \\
t=\left(m_{1}^{\dagger}\right)^{-1}=u^{(4)} \mathcal{T} u^{(1)} \\
r^{\prime}=m_{2} m_{4}^{-1}=u^{(4)} \mathcal{R} u^{(2)} \\
t^{\prime}=m_{4}^{-1}=u^{(3)} \mathcal{T} u^{(2)}
\end{gathered}
$$

where $u^{(l)}(l=1, \ldots, 4)$ are arbitrary $N \times N$ unitary matrices. $\mathcal{R}$ and $\mathcal{T}$ are real diagonal $N \times N$ matrices whose non-zero elements (labeled by only one index) are the square roots of the reflection and transmission eigenvalues which can be expressed as a function of the real positive diagonal elements $\lambda_{a}(a=1, \ldots, N)$ of the $N \times N$ diagonal matrix $\lambda$ characterizing the radial part of $M$ :

$$
\begin{gathered}
\mathcal{R}_{a}=\left(\frac{\lambda_{a}}{1+\lambda_{a}}\right)^{1 / 2} \\
\mathcal{T}_{a}=\left(\frac{1}{1+\lambda_{a}}\right)^{1 / 2} .
\end{gathered}
$$

In this $\lambda$-parametrization, $M$ and $S$ can then be written as

\footnotetext{
${ }^{3}$ Throughout this work we shall frequently represent $2 N \times 2 N$ matrices in terms of their $N \times N$ blocks. We reserve capital letters for $2 N \times 2 N$ matrices, while calligraphic and low-case letters are used for $N \times N$ matrices.
} 


$$
\begin{gathered}
M=\left(\begin{array}{cc}
u^{(4)} & 0 \\
0 & u^{(2) \dagger}
\end{array}\right)\left(\begin{array}{cc}
(I+\lambda)^{1 / 2} & \lambda^{1 / 2} \\
\lambda^{1 / 2} & (I+\lambda)^{1 / 2}
\end{array}\right)\left(\begin{array}{cc}
u^{(1)} & 0 \\
0 & u^{(3) \dagger}
\end{array}\right) \\
S=\left(\begin{array}{cc}
u^{(3)} & 0 \\
0 & u^{(4)}
\end{array}\right)\left(\begin{array}{cc}
-\mathcal{R} & \mathcal{T} \\
\mathcal{T} & \mathcal{R}
\end{array}\right)\left(\begin{array}{cc}
u^{(1)} & 0 \\
0 & u^{(2)}
\end{array}\right) .
\end{gathered}
$$

Since $t t^{\dagger}=u_{1} \mathcal{T}^{2} u_{1}^{\dagger}$, the dimensionless conductance can be expressed as

$$
g=\sum_{a=1}^{N} \frac{1}{1+\lambda_{a}} .
$$

The conductance is therefore a linear statistics of the radial parameters $\left\{\lambda_{a}\right\}$ of $M$. Note that such a simple relationship does not exists between $g$ and the scattering phase-shifts $\left\{\theta_{a}\right\}$, since the relation between the eigenvalues of $S$ and those of $t t^{\dagger}$ depends on the eigenvectors of $S$ and on the $u$-matrices.

In the absence of a magnetic field there is time reversal symmetry, the $S$-matrix is symmetric $\left(S=S^{\mathrm{T}}\right)$ and the polar decomposition has only two arbitrary unitary matrices since

$$
\begin{aligned}
& u^{(3)}=u^{(1) \mathrm{T}}, \\
& u^{(4)}=u^{(2) \mathrm{T}} .
\end{aligned}
$$

In this case the number of independent parameters is to $2 N^{2}+N$. We have $N^{2}$ parameters for each of the two $N \times N$ unitary matrices and $N$ for the diagonal matrix $\lambda$. Without time-reversal symmetry (unitary case with spin degeneracy) the number of independent parameters of $S$ (and $M$ ) is $4 N^{2}$ (the $N$ extra parameters of the polar decompositions (2.9) and (2.8) are due to the fact that they are not unique [15]). In the symplectic case occuring when there is a strong spin-orbit scattering in the disordered part and no applied magnetic field, the spin degenracy is removed and each matrix element becomes a $2 \times 2$ quaternion matrix, which doubles the size of $M$ and $S$, but $u^{(3)}$ and $u^{(4)}$ are also given [22] in terms of $u^{(1)}$ and $u^{(3)}$ and the $\lambda$ have a twofold degeneracy (Kramers degeneracy).

\section{INVARIANT MEASURE OF $S$ IN THE POLAR DECOMPOSITION}

In this section we calculate the invariant measure $\mu(d S)$ of $S$ in terms of the radial parameters $\left\{\lambda_{a}\right\}$ and the matrices $u^{(l)}$. We present here the time-symmetric case $(\beta=1)$, while the unitary case $(\beta=2)$ relevant when a magnetic field is applied is considered in Appendix D. Our calculations have recently been extended by K. Frahm [23 for the symplectic case $(\beta=4)$. In the orthogonal case $S$ is unitary symmetric and can be decomposed as:

$$
S=W^{\mathrm{T}} \Sigma W=U^{\mathrm{T}} \Gamma U=Y^{\mathrm{T}} Y .
$$

The first equality simply means the diagonalization of $S$ and introduces the $2 N$ phase shifts of $S$ through the diagonal elements $\exp \left(i \theta_{m}\right)$ of $\Sigma$ and a $2 N \times 2 N$ orthogonal matrix $W$ 
containing the eigenvectors of $S$. The second equality results of the polar representation of $S$ where the real matrix $\Gamma$ and the block-diagonal unitary matrix $U$ are given by Eq. (2.9) with the conditions (2.11). The last decomposition holds for any unitary symmetric matrix and introduces a $2 N \times 2 N$ unitary matrix $Y$ which is not unique, but specified up to an orthogonal transformation. Following Dyson [7], the measure of a neighbourhood $d S$ of $S$ is given in terms of the infinitesimal variations $d \tilde{M}_{i j}$ of a the matrix elements of a real symmetric matrix $d \tilde{M}$ defined by:

$$
\begin{gathered}
d S=Y^{\mathrm{T}}(i d \tilde{M}) Y \\
\mu(d S)=\prod_{i \leq j}^{2 N} d \tilde{M}_{i j} .
\end{gathered}
$$

This definition is independent of the particular choice of the unitary matrix $Y$ and we use this freedom of choice to take a convenient $Y$ for expressing $d \tilde{M}$ in the $\lambda$-parametrization. To this end, we note that $\Gamma$ is real symmetric and unitary, with eigenvalues \pm 1 and diagonalizable by an orthogonal transformation $O$ :

$$
\begin{gathered}
\Gamma=O^{\mathrm{T}} D O \\
D=\left(\begin{array}{cc}
I & 0 \\
0 & -I
\end{array}\right) \quad, \quad O=\left(\begin{array}{cc}
\mathcal{P} & \mathcal{Q} \\
\mathcal{Q} & -\mathcal{P}
\end{array}\right) .
\end{gathered}
$$

The $N \times N$ blocks of $O$ are diagonal matrices given by

$$
\mathcal{P}_{a}=\frac{1}{\sqrt{2}} \sqrt{1-\mathcal{R}_{a}} \quad, \quad \mathcal{Q}_{a}=\frac{1}{\sqrt{2}} \sqrt{1+\mathcal{R}_{a}}
$$

Writing the diagonal matrix $D$ as $F^{2}$, with

$$
F=\left(\begin{array}{rr}
I & 0 \\
0 & i I
\end{array}\right)
$$

one can write $S$ as $Y^{\mathrm{T}} Y$, with

$$
Y=F O U=\left(\begin{array}{lr}
\mathcal{P} u^{(1)} & \mathcal{Q} u^{(2)} \\
i \mathcal{Q} u^{(1)} & -i \mathcal{P} u^{(2)}
\end{array}\right) .
$$

Since $Y$ is unitary, its infinitesimal variations can be expressed as

$$
d Y=\delta Y Y
$$

where the matrix $\delta Y$ is antihermitic. Analogously, for the bock components of $U$ we can write

$$
d u^{(l)}=\delta u^{(l)} u^{(l)}, \quad \delta u^{(l)}=d a^{(l)}+i d s^{(l)}, \quad l=1,2 .
$$


$d a^{(l)}\left(d s^{(l)}\right)$ are real antisymmetric (symmetric) $N \times N$ matrices. The Haar measure $\mu\left(d u^{(l)}\right)$ for the unitary matrices $u^{(l)}$ satisfies $\mu\left(d u^{(l)}\right)=\prod_{a} d s_{a a}^{(l)} \prod_{a<b} d a_{a b}^{(l)} d s_{a b}^{(l)}$. Therefore the infinitesimal variations of $Y$ and $S$ are given by

$$
\begin{gathered}
\delta Y=\left(\begin{array}{cc}
0 & i(d \mathcal{Q} \mathcal{P}-d \mathcal{P} \mathcal{Q}) \\
i(d \mathcal{Q} \mathcal{P}-d \mathcal{P} \mathcal{Q}) & 0
\end{array}\right)+ \\
+\left(\begin{array}{cc}
\mathcal{P} \delta u^{(1)} \mathcal{P}+\mathcal{Q} \delta u^{(2)} \mathcal{Q} & i\left(-\mathcal{P} \delta u^{(1)} \mathcal{Q}+\mathcal{Q} \delta u^{(2)} \mathcal{P}\right) \\
i\left(\mathcal{Q} \delta u^{(1)} \mathcal{P}-\mathcal{P} \delta u^{(2)} \mathcal{Q}\right) & \mathcal{Q} \delta u^{(1)} \mathcal{Q}+\mathcal{P} \delta u^{(2)} \mathcal{P}
\end{array}\right) \\
d S=Y^{\mathrm{T}}\left(\delta Y-\delta Y^{*}\right) Y=Y^{\mathrm{T}}(i d \tilde{M}) Y
\end{gathered}
$$

This give us the real symmetric matrix $d \tilde{M}$ in terms of the radial parameters $\left\{\lambda_{a}\right\}$, the unitary matrices $u^{(l)}$ and their infinitesimal variations $\left\{d \lambda_{a}\right\}$ and $\delta u^{(l)}$. We just need to calculate a Jacobean which can be decomposed as the product of three determinants,

$$
\begin{gathered}
\prod_{a=1}^{N} d \tilde{M}_{a, a+N}=\prod_{a=1}^{N} \frac{1}{2 \sqrt{\lambda_{a}}\left(1+\lambda_{a}\right)} d \lambda_{a} \\
\prod_{a<b}^{N} d \tilde{M}_{a, b+N} d \tilde{M}_{b, a+N}=\prod_{a<b}^{N} 2\left(\sqrt{\frac{\lambda_{a}}{\lambda_{a}+1}}-\sqrt{\frac{\lambda_{b}}{\lambda_{b}+1}}\right) d a_{a b}^{(1)} d a_{a b}^{(2)} \\
\prod_{a \leq b}^{N} d \tilde{M}_{a, b} d \tilde{M}_{a+N, b+N}=\prod_{a=1}^{N} 4 \sqrt{\frac{\lambda_{a}}{\lambda_{a}+1}} d s_{a a}^{(1)} d s_{a a}^{(2)} \prod_{a<b}^{N} 2\left(\sqrt{\frac{\lambda_{a}}{\lambda_{a}+1}}+\sqrt{\frac{\lambda_{b}}{\lambda_{b}+1}}\right) d s_{a b}^{(1)} d s_{a b}^{(2)},
\end{gathered}
$$

which eventually gives for the invariant measure of the symmetric unitary matrix $S$

$$
\mu(d S)=\prod_{a=1}^{N} \frac{1}{\left(1+\lambda_{a}\right)^{3 / 2}} \prod_{a, b}^{N}\left|\frac{1}{1+\lambda_{a}}-\frac{1}{1+\lambda_{b}}\right| \mu(d \lambda) \prod_{l=1}^{2} \mu\left(d u^{(l)}\right)
$$

in terms of the measure $\mu(d \lambda)=\prod_{a=1}^{N} d \lambda_{a}$ of the matrix $\lambda$ and of the Haar measures $\mu\left(d u^{(l)}\right)$ of the matrices $u^{(l)}$.

\section{DYSON CIRCULAR ENSEMBLES AND QUASI- $1 D$ DISORDERED SYSTEMS}

For the Dyson circular ensembles the number of $S$-matrices in a volume element $d S$ of measure $\mu(d S)$ around a given $S$ is just proportional to $\mu(d S)$ :

$$
P(d S)=\frac{1}{V} \mu(d S),
$$


$V$ is a normalization constant. Using the $\lambda$-parametrization, one obtains that the matrices $u^{(l)}$ are independent from each other (except by symmetry relations) and distributed according the invariant Haar measure on the unitary group, while the $N$ parameters $\lambda_{a}$ are statistically independent from the $u$-matrices and have a joint probability distribution which can be expressed in the usual Coulomb gas analogy as a Gibbs function

$$
P\left(\left\{\lambda_{a}\right\}\right)=\exp \left(-\beta \mathcal{H}\left(\left\{\lambda_{a}\right\}\right)\right)
$$

The symmetry parameter $\beta$ plays the role of an inverse temperature and the effective hamiltonian $\mathcal{H}$ is characterized by a logarithmic pairwise interaction and a one-body potential:

$$
\begin{gathered}
\mathcal{H}\left(\left\{\lambda_{a}\right\}\right)=\sum_{a<b}^{N} f\left(\lambda_{a}, \lambda_{b}\right)+\sum_{a=1}^{N} V\left(\lambda_{a}\right) \\
f\left(\lambda_{a}, \lambda_{b}\right)=-\log \left|\lambda_{a}-\lambda_{b}\right| \\
V(\lambda)=\left(N+\frac{\beta-2}{2 \beta}\right) \log (1+\lambda) .
\end{gathered}
$$

This Coulomb gas analogy characterizes the orthogonal, unitary and symplectic ensembles which differ not only by the value of the "temperature" $\beta^{-1}$, but also by the presence of a small $\beta$-dependent correction to the leading behavior of $V(\lambda)$ in a large $N$-expansion. It is remarkable that the pairwise interaction for the $\lambda$-parameter is the same than in the global maximun entropy approach to the transfer matrix [15], while $V(\lambda)$ differs in two important aspects: it is essentially proportional to $\log \lambda$ instead of $\log ^{2} \lambda$ [24] for large values of $\lambda$, and the prefactor is just the number of modes $N$ instead of the classical conductance $N l / L_{x}$. These differences are not surprising since forward and backward scattering are essentially put on the same footing in the the circular ensembles, leading to a total transmission intensity of the order of the total reflection intensity, $T \approx R \approx N / 2$ (up to weak-localization corrections) [17,18. For a disordered conductor or insulator, the refection $R$ is much larger than the transmission $T$. Clearly, bulk diffusion characterized by an elastic mean-free-path $l$ cannnot be described by the circular ensembles, which are appropriate for systems where an injected carrier is subjected to a chaotic dynamics before finding [with equal chance] one of the two injection leads. However, as shown by Beenakker [25], in the large $N$-limit, the $\lambda$ density-density correlation function (and therefore the variance of any linear statistics like the conductance) depends only on the pairwise interaction, and not on the particular form for $V(\lambda)$. Consequently, both the circular ensembles and the global maximum entropy approach to the transfer matrix yield identical UCF [26] values $2 /(16 \beta)$, slightly different from the perturbative microscopic result [2, 3] for quasi-1 $d$ disordered conductors $2 /(15 \beta)$.

In the polar representation of $S$, one can precisely see the difference between the circular ensembles and those appropriate for quasi-one dimensional disordered systems. For this, we just need to recall what we know from another statistical approach introduced for arbitrary $N$ by Dorokhov [27] from microscopic considerations and by Mello et al [28,21] from a maximum entropy assumption for the infinitesimal transfer matrix of the building block of 
a quasi-1d series. These works are based on an isotropy hypothesis: it is assumed that the $u$-matrices are distributed with the Haar measure on the unitary group and statistically independent from the radial part of $M$ (see Eq. 2.8). This limits their conclusions to quasi-one dimension and yields a Fokker-Planck equation for $P\left(\left\{\lambda_{a}\right\}\right)$ which implies the same UCF and weak-localization corrections for quasi-1d conductors than those given by diagrammatic calculations. The evolution of $P\left(\left\{\lambda_{a}\right\}\right)$ with the length $L_{x}$ of the disordered part is given by an heat equation where the Laplacian becomes the radial part of the LaplaceBeltrami operator on a space of negative curvature. Using Sutherland's transformation, Beenakker and Rejaei [29] have mapped this diffusion equation into a Schrödinger equation (with imaginary time) of a quantum set of point like particles free to move on a half line (the positive part of the real axis) within a certain potential. For arbitrary values of $\beta$, these particles have a pairwise interaction, attractive for $\beta=1$ and repulsive for $\beta=4$, making difficult to find the solution. Fortunately, this interaction vanishes for $\beta=2$, and the solution of the diffusion equation is reduced to an exactly solvable quantum $N$-body free fermion problem. This gives for the unitary case a pairwise interaction

$$
f\left(\lambda_{a}-\lambda_{b}\right)=-\frac{1}{2} \ln \left|\lambda_{a}-\lambda_{b}\right|-\frac{1}{2} \ln \left|\operatorname{arcsinh}^{2}\left(\sqrt{\lambda_{a}}\right)-\operatorname{arcsinh}^{2}\left(\sqrt{\lambda_{b}}\right)\right|,
$$

which reduces to the usual logarithmic interaction assumed by the global approach for $\left|\lambda_{a}-\lambda_{b}\right| \ll 1$, but which is halved if $\left|\lambda_{a}-\lambda_{b}\right| \gg 1$ in the quasi-1d diffusive or localized limit. This discrepancy is responsible for the slightly different UCF values characterizing ballistic quantum dots with chaotic dynamics and quasi-1d disordered conductors.

In the localized regime, the global and local approaches give identical symmetry dependence of the localization lengths, though the (log) conductance fluctuations differs by a factor 2 in the quasi-1d localized limit [30]. This later point again is consistent with the halving of the pairwise interaction $f\left(\lambda_{a}-\lambda_{b}\right)$ for large eigenvalue separations given by the local approach. For metals and insulators far from a quasi-one dimensional shape, a more dramatic shrinkage of the validity of the universal RMT-correlations has been observed [24]. This means that transverse diffusion (or even more transverse localization [31]) yields a more significant reduction of the RMT pairwise interaction than the one obtained in quasi-one dimension by Beenakker and Rejaei. To address this problem, we return to the study of the more familiar scattering phase shifts of $S$.

\section{SCATTERING PHASE SHIFTS IN QUASI-1D METALS AND A SINGLE CIRCULAR ENSEMBLE.}

As discussed in the previous section, when applied to quasi-1d conductors, the circular ensembles give the right statistics for the $u$-matrices, and locally the correct interaction $f\left(\lambda_{a}, \lambda_{b}\right)$, but certainly not the appropriate confining potential $V(\lambda)$. Using now the eigenvalue-eigenvector parametrization of $S$, we study the phase shift distribution in the case of weak disorder and quasi-1d samples. We first introduce the basic notation, then write the expected universal correlation for Dyson ensembles, which we compare to our numerical results.

Since the $S$-matrix is unitary, its $2 N$ eigenvalues are given by $2 N$ phase shifts $\theta_{m}$. Following Blümel and Smilansky [19] we write the phase shift density as 


$$
\rho(\theta)=\sum_{m=1}^{M}\left\langle\delta\left(\theta-\theta_{m}\right)\right\rangle=\frac{1}{2 \pi} \sum_{n=-\infty}^{\infty} \exp (-i n \theta)\left\langle\operatorname{Tr} S^{n}\right\rangle
$$

where the angular brackets indicate average over the ensemble of disordered samples and $M=2 N$ is the dimension of $S$. The two-point correlation function $R_{2}$ is defined by

$$
R_{2}\left(\theta_{1}, \theta_{2}\right)=\sum_{m \neq m^{\prime}}^{M}\left\langle\delta\left(\theta_{1}-\theta_{m}\right) \delta\left(\theta_{2}-\theta_{m^{\prime}}\right)\right\rangle
$$

When the phase shift distribution is uniform $(\rho(\theta)=M / 2 \pi)$ the two-point correlation function depends only on the difference $\eta=\theta_{2}-\theta_{1}$,

$$
R_{2}(\eta)=\frac{M}{(2 \pi)^{2}} \sum_{n=-\infty}^{\infty}\left(\frac{1}{M}\left\langle\left|\operatorname{Tr} S^{n}\right|^{2}\right\rangle-1\right) \exp (\text { in } \eta) .
$$

The two-level cluster function is defined, for the reduced variable $r=\eta M / 2 \pi$, in the limit where the number of phases $M$ goes to infinity, as:

$$
\begin{gathered}
Y_{2}(r)=\lim _{M \rightarrow \infty} \hat{Y}_{2}^{M}(r) \\
\hat{Y}_{2}^{M}(r)=\left(\frac{2 \pi}{M}\right)^{2}\left(\left(\frac{M}{2 \pi}\right)^{2}-R_{2}\left(\frac{2 \pi r}{M}\right)\right) .
\end{gathered}
$$

Using the expression (5.3) we have that

$$
\hat{Y}_{2}^{M}(r)=\frac{1}{M}\left(1-2 \sum_{n=1}^{\infty} s_{n}^{M} \cos \left(\frac{2 \pi n r}{M}\right)\right),
$$

where Fourier components $s_{n}^{M}$ are given by

$$
s_{n}^{M}=\frac{1}{M}\left\langle\left|\operatorname{Tr} S^{n}\right|^{2}\right\rangle-1
$$

The argument $r$ of the cluster function goes from $-\infty$ to $+\infty$, and the Fourier transform of $Y_{2}$, the two-level form factor (TLFF), is given by

$$
b(k)=\int_{-\infty}^{\infty} d r Y_{2}(r) \exp (2 \pi i k r)
$$

Comparing the Fourier transform of $Y_{2}$ and the Fourier coefficients of $\hat{Y}_{2}^{M}$ in the large $M$ limit, we can identify

$$
s_{n}^{M} \approx-b(n / M) \quad, \quad M \gg 1 .
$$

For matrices $S$ belonging to Dyson ensembles, the distribution of phase shifts is given by the Coulomb gas analogy: 


$$
P\left(\left\{\theta_{a}\right\}\right)=\frac{1}{Z} \exp \left(-\beta \mathcal{H}\left\{\theta_{a}\right\}\right)
$$

where the effective hamiltonian is

$$
\mathcal{H}\left(\left\{\theta_{a}\right\}\right)=-\sum_{a<b}^{M} \log \left|e^{i \theta_{a}}-e^{i \theta_{b}}\right| .
$$

The cluster functions of the circular ensembles have universal forms which depends only on the system symmetries. For the unitary and orthogonal ensembles $Y_{2}$ is an even function of its argument and has the form [9] f]

$$
\begin{gathered}
Y_{2}^{U E}(r)=\left(\frac{\sin \pi r}{\pi r}\right)^{2}, \\
Y_{2}^{O E}(r)=\left(\frac{\sin \pi r}{\pi r}\right)^{2}-(\operatorname{Si}(\pi r)-\pi \varepsilon(r))\left(\frac{\cos \pi r}{\pi r}-\frac{\sin \pi r}{(\pi r)^{2}}\right) .
\end{gathered}
$$

The corresponding form factors are also even functions of their argument, and have the universal forms:

$$
\begin{gathered}
b_{U E}(k)=\left\{\begin{array}{ll}
1-k & \text { if } k \leq 1 \\
0 & \text { if } k \geq 1
\end{array},\right. \\
b_{O E}(k)=\left\{\begin{array}{ll}
1-2 k+k \ln (1+2 k) & \text { if } k \leq 1 \\
1-k \ln \left(\frac{2 k+1}{2 k-1}\right) & \text { if } k \geq 1
\end{array} .\right.
\end{gathered}
$$

We check for a quasi-1d metal (Fig. 2) the agreement between the numerically generated Fourier components $s_{n}^{M}$ and the universal two-level form factors $b(k)$ of Eq. (5.13), assuming Eq. (5.9). The $S$-matrix of disordered strips described by a tight-binding Anderson model of $34 \times 136$ sites (details given in Appendix A) are numerically evaluated. Averaging involves 5000 different impurity configurations. The Fermi energy in units of the constant off-diagonal hopping term is $E=-2.5$, the number of propagating modes is $N=14$ and therefore the dimension of $S$ is $M=28$. A relatively low wave-vector ( $k=1.32$ in Anderson units) is taken in order to avoid lattice effects, since we will be interested in the comparison between our simulations and analytical approaches (diagrammatic perturbation theory and semiclassical approximation) assuming a continuum limit. One finds a rather good agreement for the time-reversal symmetric case (run R1, no magnetic field, COE-like) and for the non timereversal symmetric case (run F1, with magnetic field, CUE-like). The distribution of the phase shifts (inset) is quite uniform with and without magnetic field. The disorder in the

\footnotetext{
${ }^{4}$ We follow the standard notation: $\operatorname{Si}(x)=\int_{0}^{x} \frac{\sin y}{y} d y ; \operatorname{Ci}(x)=\mathcal{C}+\ln x+\int_{0}^{x} \frac{\cos y-1}{y} d y ; \epsilon(x)=$ $-1 / 2,0,1 / 2$, for $x<0, x=0, x>0$ respectively; $\mathcal{C}=0.5772 \ldots$ is the Euler constant.
} 
samples is very weak ( $W=1$ in units of the hopping term) giving an elastic mean-free-path $l=0.7 L_{y}$, and an average conductance $\langle g\rangle=4.1$.

We conclude that the phase shift density and correlations for a quasi-1d conductor are well approximated by the corresponding COE-CUE density and correlations. Since it is clear in the $\lambda$-parametrization that a quasi-1d conductor cannot be seen as a member of a COE-CUE ensemble, we suspect that this numerical result merely indicates a good approximation, and that the main non COE-CUE behavior of $S$ for a quasi-1d conductor must occur in the distribution of its eigenvectors. As we will see in the following sections, the good agreement with the universal correlations gets poorer as we increase the disorder, enter into the quasi-1d localized regime, or go outside the quasi-1d geometry.

\section{SCATTERING PHASE SHIFTS IN QUASI-1 $D$ INSULATORS AND TWO UNCOUPLED CIRCULAR ENSEMBLES.}

The approximate COE-CUE behavior which we found in the previous section cannot remain in the presence of quasi-1d localization for obvious reasons: $g \ll 1$ and a typical matrix element of the reflection matrices $r$ and $r^{\prime}$ is much larger than those of $t$ and $t^{\prime}$. The matrix $S$ can then be thought as two diagonal blocks, $r$ and $r^{\prime}$, weakly coupled by $t$ and $t^{\prime}$. Using the polar decomposition, Eq. (2.9), and the fact that the radial parameters $\left\{\lambda_{a}\right\}$ are exponentially large in the localized regime, one can write

$$
\begin{gathered}
r=-u^{(3)} u^{(1)}+\mathcal{O}\left(\lambda^{-1}\right), \\
r^{\prime}=u^{(4)} u^{(2)}+\mathcal{O}\left(\lambda^{-1}\right) .
\end{gathered}
$$

A strong quasi-1d localization implies that $S$ reduces to two uncoupled $N \times N$ unitary (symmetric if the presence of time reversal symmetry) matrices and isotropy means that each of them is invariant under orthogonal (unitary) transformation. The phase shifts associated to $r$ and $r^{\prime}$ will then be described separately by two uncoupled COE-CUE ensembles. For a weaker quasi-1d localization, we have a cross-over behavior between a set of $2 N$ phase shifts with approximately COE-CUE correlations to two uncoupled sets of $N$ exactly COECUE phase shifts. We underline that the observed COE-CUE phase shift distribution for the quasi-1d conductor is less trivial than the COE-CUE character of the reflection matrices for strong quasi-1d localization which only results from the isotropy assumption. A similar decoupling of the $S$-matrix in two nearly independent blocks have also been discussed recently by Borgonovi and Guarneri [33.

If $Y_{2}(r)$ and $b(k)$ are the two-level cluster function and the two-level form factor of two independent ensembles, the corresponding functions for the combined ensemble are given by 32

$$
\begin{gathered}
Y_{2}^{s}(r)=\frac{1}{2} Y_{2}(r / 2), \\
b_{s}(k)=b(2 k) .
\end{gathered}
$$


This expected crossover situation towards two uncoupled COE-CUE, for sample lengths of order of the localization length, is indeed observed in Fig. 3 where we show the Fourier components $s_{n}^{M}$ with and without magnetic field. For the very long, weakly disordered samples (R10, diamonds, $A R=30, W=1, N=14$ ) the phase shift distribution (inset) is as homogeneous as for the quasi-1d conductor. The low harmonics of $Y_{2}$ (small $n$ values of $s_{n}^{M}$ ) behave as those of two uncoupled COE-CUE, reflecting the statistical independence of the short length trajectories contributing to reflection. Higher order harmonics behave more like a single $\mathrm{COE}-\mathrm{CUE}$, indicating that localization is not sufficient for decoupling the long trajectories. For a better understanding of this effect, one can use the semiclassical picture developed in Appendix Q, where for relatively small values of $n, s_{n}^{M}$ is given in terms of periodic orbits of the closed sample which hit $n$ times the vertical limiting hard walls: small values of $n$ are related with short reflection trajectories which stay close to each of the samples edges and do not explore the other extreme of the sample (two decoupled COE-CUE behavior). On the contrary, for the large values of $n$, the contribution of the transmission trajectories can not be ignored, and removes the statistical independence of the two reflection matrices.

Increasing of the system length being time consuming for the numerical simulations, we can more easily achieve localization by increasing the strenght of the disorder potential keeping the geometry fixed. In this case we are able to obtain a more complete decoupling, extending to higher harmonics the characteristic behavior of two independent COE-CUE (R4-F4, filled circles, $A R=4, W=4, N=14$ ). Having stronger localization yielded by stronger disorder, we note an additional effect: the phase shift density is no longer uniform. We will discuss this departure from isotropy in detail in the next section. For the purpose of the present discussion we only indicate that we numerically unfold the phase shift spectra to a rescaled spectra of uniform density. Then, one can see in Fig. 3 a rather complete statistical decoupling of the two sample edges. Let us note also that the small $n$ behavior of $s_{n}^{M}$ is now a little above what we expect from two uncoupled COE-CUE, a point which will be considered in Sec. VIII.

In order to study more precisely how the transition from the metallic case (COE or CUE-like cases) to the localized regime takes place, we calculate the number $n(r)$ of levels contained in an interval of length $r$ for the unfolded spectum of the phase shifts and its variance (number variance)

$$
\Sigma^{2}(r)=\left\langle(n(r)-r)^{2}\right\rangle
$$

which can be obtained directly from the numerical data and compared with universal forms of the standard ensembles. Using Eq. (5.12), one gets for the unitary and orthogonal cases (9)

$$
\begin{gathered}
\Sigma_{U E}^{2}(r)=\frac{1}{\pi^{2}}(\ln (2 \pi r)+\mathcal{C}+1-\cos (2 \pi r)-\operatorname{Ci}(2 \pi r))+r\left(1-\frac{2}{\pi} \operatorname{Si}(2 \pi r)\right), \\
\Sigma_{O E}^{2}(r)=2 \Sigma_{U E}^{2}(r)+\left(\frac{\operatorname{Si}(\pi r)}{\pi}\right)^{2}-\frac{\operatorname{Si}(\pi r)}{\pi},
\end{gathered}
$$

with the large- $r$ behavior 


$$
\begin{gathered}
\Sigma_{U E}^{2}(r)=\frac{1}{\pi^{2}}(\ln (2 \pi r)+\mathcal{C}+1)+\mathcal{O}\left(r^{-1}\right), \\
\Sigma_{O E}^{2}(r)=\frac{2}{\pi^{2}}\left(\ln (2 \pi r)+\mathcal{C}+1-\pi^{2} / 8\right)+\mathcal{O}\left(r^{-1}\right) .
\end{gathered}
$$

For the superposition of two independent ensembles, one gets 32

$$
\Sigma_{s}^{2}(r)=2 \Sigma^{2}(r / 2) .
$$

Since our original phase shift spectrum is bounded between 0 and $2 \pi, \Sigma^{2}(r)$ folds back to 0 for $r=M$ as the number of phases in $0<\theta<2 \pi$ (or $0<\Theta<M$ ) is always $M$. Hence our comparisons between our numerical data and Eqs. (6.5) - 6.7) are meaningful for $r \ll M$ and we focus our attention to intervals $0<r<M / 3$.

In Fig. 4 we show how the number variance $\Sigma^{2}(r)$ changes from the metallic to the localized regime when we increase the disorder or the sample length. If the disorder is increased, we obtain a continuous transition from the COE case towards less rigid spectrums and the phase shift density develops a more and more non uniform structure. The strength of the local disorder not only decouples the right and left reflections, but also introduces in the transverse direction a dimensionality dependent dynamics, which breaks isotropy. The number variance can then exceed that of two uncoupled COE.

When the disorder is small, and localization is achieved by increasing the system length, the transverse dynamics remains essentially zero dimensional, $S$ is isotropic and the number variance indicates a cross-over from approximately a single COE for the $2 N$ phase shifts of $S$ (quasi-1d conductor) to two decoupled COE-like sets of $N$ phase shifts associated to right and left reflections.

When a magnetic field is applied, we obtain similar results, with a slightly improved agreement with the CUE-like character: the applied magnetic field suppressing coherent interferences between time reversed trajectories shifts the sample towards the metallic regime and doubles the localization length [30].

\section{AVERAGE SCATTERING MATRIX AND ISOTROPY HYPOTHESIS}

Quasi-1d distributions are partly based on the isotropy hypothesis: i.e. the unitary matrices $u^{(l)}$ are uncorrelated with the radial part of $S$ and distributed with the invariant measure on the unitary group. This implies that the ensemble average of $S$ must be zero. This property does not hold for high disorder and non quasi-1d geometry. For instance, a non uniformity of the phase shift distribution was noticed in the previous section for a large disorder and occurs for weaker disorder in shorter samples (squares and thin slabs). This source of discrepancy with the circular ensemble is studied in this section.

In the circular ensembles, the phase shift density is uniform and equal to $\rho_{0}=M / 2 \pi$. This means that $\left\langle\operatorname{Tr} S^{n}\right\rangle=0$ for all $n \neq 0$. We will see that this is not the case for disordered systems. The first harmonic of the Fourier expansion of the phase shift density $\rho(\theta)$ is:

$$
\rho_{1}(\theta)=\frac{1}{\pi} \operatorname{Re}[\exp (-i \theta)\langle\operatorname{Tr} S\rangle]
$$


and $\langle\operatorname{Tr} S\rangle=0$ is a necessary condition to obtain uniform phase shift distribution. Since

$$
\langle\operatorname{Tr} S\rangle=\sum_{a=1}^{N}\left(\left\langle r_{a a}\right\rangle+\left\langle r_{a a}^{\prime}\right\rangle\right),
$$

we can calculate $\rho_{1}(\theta)$ by evaluating the mean values of the diagonal reflection elements in perturbation theory.

The transmission (reflection) amplitude from a mode $a$ on the left to a mode $b$ on the right (left) for electrons at the Fermi energy $E_{f}=\hbar^{2} k^{2} / 2 m$ is given by [34]

$$
\begin{gathered}
t_{b a}=-i \hbar\left(v_{a} v_{b}\right)^{1 / 2} \int d y^{\prime} \int d y \phi_{b}^{*}\left(y^{\prime}\right) \phi_{a}(y) G_{k}\left(L_{x}, y^{\prime} ; 0, y\right) \\
r_{b a}=\delta_{a, b}-i \hbar\left(v_{a} v_{b}\right)^{1 / 2} \int d y^{\prime} \int d y \phi_{b}^{*}\left(y^{\prime}\right) \phi_{a}(y) G_{k}\left(0, y^{\prime} ; 0, y\right)
\end{gathered}
$$

where $v_{a}\left(v_{b}\right)$ and $\phi_{a}\left(\phi_{b}\right)$ are the longitudinal velocity and transverse wavefunction for the incoming (outgoing) mode $a(b)$. For hard-wall boundary conditions, the transverse wave functions have the sinusoidal form presented in Sec. [I, $v_{n}=\hbar k_{n} / m, k_{n}^{2}=k^{2}-\left(n \pi / L_{y}\right)^{2}$, $n=a, b$. We note by $m$ the effective mass of the electrons. For the transmission (reflection) amplitudes $G_{k}\left(\mathbf{r}^{\prime} ; \mathbf{r}\right)$ is the retarded Green function evaluated at the Fermi energy between points $\mathbf{r}=(x, y)$ on the left lead and $\mathbf{r}^{\prime}=\left(x^{\prime}, y^{\prime}\right)$ on the right (left) lead. Similar expressions hold for the transmission (reflection) amplitudes for modes comming from the right by using $G_{k}\left(0, y^{\prime} ; L_{x}, y\right)$ in Eq. (7.3a) and $G_{k}\left(L_{x}, y^{\prime} ; L_{x}, y\right)$ in Eq. (7.3b) instead of $G_{k}\left(L_{x}, y^{\prime} ; 0, y\right)$ and $G_{k}\left(0, y^{\prime} ; 0, y\right)$ (and placing the $y$ abscissa at $x=L_{x}$, and $y^{\prime}$ at $x=0\left(L_{x}\right)$ ).

For a given impurity configuration, the unaveraged retarded Green function $G_{k}$ for electrons at the Fermi level in the absence of a magnetic field satisfies

$$
\left(\frac{\hbar^{2}}{2 m} \nabla_{\mathbf{r}}^{2}+\frac{\hbar^{2} k^{2}}{2 m}+V(\mathbf{r})+i \gamma\right) G_{k}\left(\mathbf{r}^{\prime} ; \mathbf{r}\right)=\delta\left(\mathbf{r}^{\prime}-\mathbf{r}\right),
$$

with $\gamma \rightarrow 0^{+}$. We will assume that the impurity potential $V(\mathbf{r})$ is given by $N_{i}$ uncorrelated $\delta$-function scatterers (of strength $u$ ) randomly distributed in the disordered strip.

$$
V(\mathbf{r})=\sum_{\alpha=1}^{N_{i}} u \delta\left(\mathbf{r}-\mathbf{R}_{\alpha}\right) \quad\left\{\begin{array}{l}
0<R_{\alpha, x}<L_{x} \\
0<R_{\alpha, y}<L_{y}
\end{array} \quad \alpha=1, \ldots, N_{i} .\right.
$$

The standard techinque used in disordered systems is to solve Eq. (7.4) in perturbation theory and take the ensemble average at each order of the perturbation expansion [35,36]. As indicated diagramatically in Fig. 5, we merely expand to second order in the perturbation expansion.

The unperturbed Green function $G_{k}^{(0)}\left(\mathbf{r}^{\prime} ; \mathbf{r}\right)$ for an infinite strip can be expanded in the base of the transverse wavefunctions $\phi_{n}$, and the $n^{\text {th }}$ coefficient is a one-dimensional Green function with an effective wave vector $k_{n}=\sqrt{k^{2}-\left(n \pi / L_{y}\right)^{2}}\left(k_{n}\right.$ is real when $n$ corresponds to a propagating mode and pure imaginary otherwise), 


$$
G_{k}^{(0)}\left(x^{\prime}, y^{\prime} ; x, y\right)=\frac{m}{i \hbar^{2}} \sum_{n=1}^{\infty} \frac{1}{k_{n}} \exp \left(i k_{n}\left|x^{\prime}-x\right|\right) \phi_{n}\left(y^{\prime}\right) \phi_{n}(y)
$$

The inclusion of $G_{k}^{(0)}$ in (7.3b) just cancels the $\delta_{a, b}$ factor since in the absence of disorder the modes propagate without any reflection. The first and second order corrections are respectively given by

$$
\begin{aligned}
& \left\langle r_{b a}^{(1)}\right\rangle=\left\langle r_{b a}^{(1)}\right\rangle=-\delta_{a, b}\left(\frac{m u}{2 \hbar^{2}}\right) \frac{n_{i}}{k_{a}^{2}}\left(1-e^{2 i k_{a} L_{x}}\right), \\
& \left\langle r_{b a}^{(2)}\right\rangle=\left\langle r_{b a}^{(2)}\right\rangle=i \delta_{a, b}\left(\frac{m u}{2 \hbar^{2}}\right)^{2} \frac{n_{i}}{k_{a}^{2}}\left(1-e^{2 i k_{a} L_{x}}\right),
\end{aligned}
$$

where $n_{i}=N_{i} /\left(L_{x} L_{y}\right)$ is the impurity density. For the second order correction we are only giving the leading term (in the impurity parameter $u n_{i}$, and in the inverse mode number $1 / N$ ), and we have cut the sum over the internal momentum (as usually done for $\delta$-function potentials). The highly oscillating phases $e^{2 i k_{a} L_{x}}$ would be suppressed by inelastic scattering. For translationally invariant (after averaging) systems the perturbation theory is usually done in momentum representation; the first order term gives rise to a real selfenergy that merely renormalizes the Fermi energy, while the second order term gives rise to an imaginary self energy which is responsible for an exponential damping of the average real space Green function. For the reflection amplitudes from finite disordered regions, we take into account perturbation up to second order term only. This calculation neglects the multiple scattering processes characteristic of the diffusive regime. However, we are only interested in the average value of the reflection amplitude, where single scattering dominates multiple scattering, as we will see in our semiclassical approach, and Eqs. (7.7) give the main features seen in the numerical simulations. They are proportional to the strength of the disorder and show a non-zero average only for the diagonal elements of the $S$-matrix. The denominator $k_{a}$ indicates that the absence of self-averaging is more pronounced for the higher modes. The breakdown of perturbation theory for small $k_{a}$ is understandable since it is close to a threshold of complete reflection which cannot be obtained perturbatively. Clearly, those threshold effects, when new conduction channels appear $(N \rightarrow N+1)$ ), drastically limit the validity of the isotropy assumption.

Up to second order perturbation the average diagonal reflection amplitudes are

$$
\left|\left\langle r_{a a}^{(1,2)}\right\rangle\right|=|\alpha| \frac{n_{i}}{k_{a}^{2}} \sqrt{2\left(1-\cos \left(2 k_{a} L_{x}\right)\right)} \quad,
$$

where $\alpha=\left(\frac{m u}{2 \hbar^{2}}\right)\left(-1+i\left(\frac{m u}{2 \hbar^{2}}\right)\right)$. For the lowest modes Eq. (7.8) gives a correction vanishing as $1 / k^{2}$ (or $\left.\left(L_{y} / N\right)^{2}\right)$, but the correction remains important for the highest modes. On the other hand, in our numerical simulations in a lattice model, $N$ will always be finite and not very large. In Fig. 6.a we show the values of $\left|\left\langle r_{a a}\right\rangle\right|$ obtained from our numerical simulations, as a function of the mode number $a$. The average reflection amplitudes of the sample described in the Sec. V (R1, squares, $A R=4, W=1, N=14)$ are close to the functional form $1 / k_{a}$ (solid thick line) and to those of a longer sample (R8, circles, $A R=10$, 
$W=1, N=14$ ). The average value of the nondiagonal reflection amplitudes (not shown) are zero within the statistical error. Since this perturbation calculation is performed in the continuum and yields a rapidly oscillating phase we do not expect to get full agreement with the numerical simulations on a lattice. We are mainly interested in the mean behavior of the diagonal reflection elements as a function of the channel number $a$, the number of modes $N$ and the strenght of the disorder $W$. Increasing the disorder (R2, filled diamonds, $A R=4$, $W=1.5, N=14$ ) enhances the correction. Keeping the number of modes $N=14$ (Fig. 6.b) while going to a square geometry (R12, squares, $A R=1, W=1, N=14$ ) decreases the Fermi momentum and gives a larger correction for the average reflection amplitude than for the sample of Sec. $\nabla$. The correction for a square geometry is of the same order of that of a long sample (R11, circles, $A R=4, W=1, N=14$ ) with the same number of modes and cross section (same Fermi momentum). Increasing the disorder (R13, diamonds, $A R=1$, $W=1.5, N=14$ ) enhances the correction, while increasing the number of modes (R18, full triangles, $A R=1, W=1.5, N=28$ ) decreases the correction.

The mean values of the scattering matrix elements have been considered by Iida, Weidenmüller and Zuk [37] who studied the interplay between universal conductance fluctuations and the statistical properties of the hamiltonian spectrum of a disordered strip. They define the sticking probability $R_{a}=1-\left|\left\langle r_{a a}\right\rangle\right|^{2}$, measuring the weight of the fast processes (where the particle is reflected after a few scattering events in the disordered region) versus the long trajectories. Sticking probabilities smaller than 1 give corrections to the universal quasi-1D value of the variance of the conductance fluctuations, which can be calculated with the aid of Eq. (7.8).

This non-zero average of the diagonal refection elements is in disagreement with the standard maximum entropy approaches where one assumes that the matrices $u^{(l)}$ are uniformely distributed in the unitary group, giving zero average values of the $S$-matrix. Recent work by Mello and Tomsovic [38] has relaxed the isotropy assumption, making possible a non-zero mean for the transmission amplitude (diagonal in mode number and exponential in $l / L_{x}$ ). Our results show that a non-zero average for the diagonal reflection amplitudes is needed in order to describe systems outside the weak disorder and quasi-one-dimension.

In the large $N$-limit we approximate the second order perturbation calculation of $\langle\operatorname{Tr} S\rangle$ by converting the sum over modes into an integral, the phases $\exp \left(2 i k_{a} L_{x}\right)$ give rise to higher order terms in 1/N, and from Eqs. (7.7) we obtain (away from the thresholds)

$$
\left\langle\operatorname{Tr} S^{(1,2)}\right\rangle \approx \alpha \frac{n_{i} L_{y}}{k \pi} \ln \left(\frac{k+N \pi / L_{y}}{k-N \pi / L_{y}}\right) \approx \alpha \frac{n_{i} L_{y}^{2}}{\pi^{2}} \frac{1}{N} \ln (2 N) .
$$

It is important to notice that Eq. (7.9) does not depend on the length $L_{x}$ of the sample (or the aspect ratio) but only depends on the properties at the entrance of the sample (transverse cross-section $L_{y}$ and Fermi momentum $k$ ). Its logarithmic dependence on $N$ indicates that there is homogeneity in the phase distribution only for very large $N$ and very small transverse cross section.

From Eqs. (7.1) and (7.9) we can see that the phase shift distribution will not be uniform, unless we have a narrow sample with weak disorder and a large number of propagating modes. A non uniform density is often the case for hamiltonian spectra (or for the radial parameters of $M$ ), and one studies the correlations of the unfolded spectra [11,39. Instead of studying 
the bare phase shifts $\theta_{m}$, we have to consider the rescaled variable $\Theta_{m}=\mathcal{N}\left(\theta_{m}\right)$, where $\mathcal{N}(\theta)$ is the number of levels below $\theta$, i.e. $\mathcal{N}(\theta)=\int_{0}^{\theta} \rho\left(\theta^{\prime}\right) d \theta^{\prime}$. Since our interval of phase shifts is bounded between 0 and $2 \pi$, for the numerical study, we repeat twice each phase shift sequence and calculate the two-point correlation function in the second interval ( $M$ to $2 M$ for the unfolded variables $\left.\Theta_{m}\right)$. The Fourier components $s_{n}^{M}$ can then be compared with the universal two-level form factors of Eq. (5.13), as we do in the following sections.

\section{UNFOLDED PHASE SHIFT SPECTRA OUTSIDE QUASI-ONE-DIMENSION}

We now turn our attention to samples far from a quasi-1d shape. For disordered squares (Fig. 7.a) the phase shift densities are non uniform in the absence of magnetic field (inset). The degree of nonuniformity is consistent with Eqs. (7.1) and (7.9), i.e. it becomes more important for higher disorder and smaller number of propagating channels. The Fourier components $s_{n}^{M}$ (after unfolding) are in relatively good agreement with the COE-CUE twolevel form factors, but the correspondence becomes poorer when we increase the disorder. For the large- $n$ Fourier components, we are probing the correlations for small separations, where we can take the phase shift density as constant. This approximate translational invariance makes that only the term with $n=-n^{\prime}$ survives in the expansion of the delta functions of Eq. (5.2). The Fourier components of the two-point correlation function are still given by $\left\langle\left|\operatorname{Tr} S^{n}\right|^{2}\right\rangle$, through Eq. (5.7). For squares with magnetic field and low disorder the phase shift density is relatively uniform (lower histogram of the inset) and we use Eq. (5.7) instead of unfolding the spectrum.

In Fig. 7.b, a disordered slab is considered (upper inset, the incoming electron flux is along the direction of the shortest dimension). The phase shift distributions (inset) are strongly non uniform ( $L_{y}$ in Eq. (7.9) is very large), but the unfolded spectra, with (triangles) and without (filled squares) magnetic field, are relatively well described by Eq. (5.13), except for small $n$ values where a careful look indicates values above the COE-CUE behavior. This is another important source of departure from the universal behavior related to transverse diffusion, in addition to the cross-over mentioned in section $\nabla 1$ coming from longitudinal localization.

The approximate agreement of the numerical data with the two-point form factors of the circular ensembles is somewhat surprising in these geometries. Given the approximate agreement of the unfolded spectra with the standard ensembles at the level of the twopoint correlation function, we might ask at this stage whether the unfolded phase shift spectrum of metallic conductors is well described by the circular ensembles, independently of the shape and strenght of the disorder. However, a check at the level of the two-point correlation function is not very accurate, as we have learned from statistical studies of chaotic hamiltonians [11] and transmission matrices [24]. A better test is provided by integrals involving the two-point correlation function. As in Sec. V1, we now consider the number statistics $n(r)$ and the number variance (Eq. (6.4))

In order to analyze systematic departures from the random matrix correlations we plot in Fig. 8, for various geometries, degrees of disorder and number of propagating modes, the difference of the number variances between the numerical data and the COE-CUE 
values (6.5). We consider $\sigma^{2}(r)=\Sigma^{2}(r)-\Sigma_{O E}^{2}(r)$ for samples without magnetic field and $\sigma^{2}(r)=\Sigma^{2}(r)-\Sigma_{U E}^{2}(r)$ for samples with nonzero magnetic field. Like in the previous section, we only show the interval $0<r<M / 3$ where the comparission is meaningful. One can see very clearly now that, even after unfolding, the accuracy of the random matrix description strongly depends on the shape and degree of disorder of the samples. The difference $\sigma^{2}(r)$ in number variances grows approximate linearly with $r$. The magnitude of the slope of $\sigma^{2}(r)$ measures the validity of the random matrix description. Squares with low disorder (after unfolding) are well represented by the standard ensembles, the agreement becomes poorer when increasing the disorder, and improves when augmenting the number of modes $N$. However, slab-shaped samples show also large deviations respect to the COE-CUE values. The conditions for having a good COE-CUE distribution coincide with the conditions for diminishing $|\langle\operatorname{Tr} S\rangle|$ and obtaining a more uniform distribution (Eqs. (7.1) and (7.9)). Notice however that Fig. 8 is done over unfolded spectra, where the nonhomogeneous phase shift distribution is in principle already accounted for. Our task in the remaining section is to to quantitatively study the deviations to the random matrix behaviors in disordered conductors, giving the slope of the curves $\sigma^{2}(r)$ as a function of the aspect ratio, degree of disorder and number of propagating modes of the disordered sample.

\section{DEVIATIONS FROM THE UNIVERSAL RMT BEHAVIOR}

The statistical properties of the spectra of small disordered systems have been considerably more studied than those of the scattering phase shifts. It is then appropriate to establish a connection between the two in order to understand our numerical results. The relationship between phase shifts and energy levels has been investigated in the semiclassical limit by Bogomolny [40] and by Doron and Smilansky [41]. Their approach is not completely applicable to our case but provides some guiding concepts. We reproduce in Appendix B their main results and discuss the points where the correspondence does not hold.

Under the assumptions discussed in Appendix B of neglecting the evanescent modes and the nonuniformity of the phase shift distribution, the energy level density is given by

$$
\mathrm{d}(E)=\frac{M}{2 \pi \hbar}\left\langle\tau_{W}(E)\right\rangle
$$

where $\tau_{W}(E)$ is the Wigner time

$$
\tau_{W}(E)=\frac{\hbar}{i M} \operatorname{Tr}\left(S^{\dagger}(E) \frac{d S(E)}{d E}\right),
$$

whose physical interpretation is discussed in Appendices 9 and B. Assuming that the density of states of our disordered rectangular samples is the same as without disordered $\mathrm{d}(E)=1 / \Delta=m L_{x} L_{y} / 2 \pi \hbar^{2}$, we can check the validity of Eq. (9.1)) against our numerical simulations. As indicated in Table I of Appendix A there is agreement within 5 to $25 \%$ in most of the cases, which is reasonable given the various approximations involved.

The quantity most often calculated in metallic spectra is the density-density correlation function 


$$
K_{2}(E, E+\varepsilon)=\sum_{n, n^{\prime}}\left\langle\delta\left(E-E_{n}\right) \delta\left(E+\varepsilon-E_{n^{\prime}}\right)\right\rangle-\mathrm{d}(E) \mathrm{d}(E+\varepsilon)
$$

Scaling the energy separation with the mean level spacing $(e=\varepsilon \mathrm{d}(E))$ and the phase shift separation with the mean phase shift distance $(r=\eta M / 2 \pi)$ we have, from Eq. (B8), in the large $M$ limit that

$$
K_{2}(e / \mathrm{d}(E)) \mathrm{d}^{-2}(E)=\delta(r)-Y_{2}(r)
$$

As stated before, we will put aside the fact that the two above mentioned assumptions are not quite true in our systems and we will pursue the consequences of Eq. (9.4).

The density-density correlation function for disordered systems has been obtained in perturbation theory by Altshuler and Shklovskiu [4]

$$
K_{2}(\varepsilon)=-\frac{s^{2}}{\pi^{2}} R e \sum_{\left\{n_{\mu}\right\}}\left(\varepsilon+i \hbar D q^{2}+i \gamma\right)^{-2},
$$

for energies $\varepsilon$ large compared to the level spacing $\Delta$, and small compared with the energy scale $\hbar / \tau_{e}$, associated with the elastic scattering time $\tau_{e}$. The factor $s$ accounts for the spin degeneracy of each level ( $s=1$ since we work with spinless electrons), and $\gamma$ is a small energy cutoff (to account for inelastic scattering). For simplicity we will be work the case of zero magnetic field. The sum is over the diffusion modes in the sample, assumed to be a $d$-dimensional parallelepiped with sides $L_{\mu}$, that is, $q^{2}=\pi^{2} \sum_{\mu=1}^{d}\left(n_{\mu} / L_{\mu}\right)^{2}$. The diffusion coefficient is $D=v_{f} l / d$. The Thouless energy $E_{c, \mu}=\hbar D / L_{\mu}^{2}$ is inversely proportional to the time that takes an electron to diffuse across the sample in the $\mu$-direction. For samples with all $L_{\mu}$ equal (hypercubes) we just have one Thouless energy. That will be the case of our square samples, while for quasi-one-dimensional samples we have in principle two Thouless energies, but we will reserve this name for the smaller one, that is, the one associated with the length $L_{x}$.

The mean square fluctuation in the number of levels (number variance) is given in terms of the density-density correlation function,

$$
\left\langle[\delta N(\varepsilon)]^{2}\right\rangle=\int_{E-\varepsilon / 2}^{E+\varepsilon / 2} d E_{1} \int_{E-\varepsilon / 2}^{E+\epsilon / 2} d E_{2} K_{2}\left(E_{1}, E_{2}\right)
$$

which from (9.5) can be written as

$$
\left\langle[\delta N(\varepsilon)]^{2}\right\rangle=\frac{1}{\pi^{2}} \sum_{\left\{n_{\mu}\right\}} \ln \left(\frac{\varepsilon^{2}}{\left(\gamma+\hbar D q^{2}\right)^{2}}+1\right) .
$$

For energies $\varepsilon \ll E_{c, \mu}$ the sum (9.7) is dominated by the term with all $n_{\mu}$ null and [⿴囗十

$$
\left\langle[\delta N(\varepsilon)]^{2}\right\rangle=\frac{1}{\pi^{2}} \ln \left(\frac{\varepsilon^{2}}{\gamma^{2}}+1\right) .
$$

The perturbation theory of Ref. [4] is valid for energy separations $\varepsilon$ larger than the inelastic scattering $\gamma$ or the level spacing $\Delta$. In our case there is no inelastic scattering 
and we substitute $\gamma$ by $\Delta$ obtaining the standard random matrix theory result $\left\langle[\delta N(\varepsilon)]^{2}\right\rangle=$ $2 / \pi^{2} \ln (\varepsilon / \Delta)$ for $\Delta \ll \varepsilon \ll E_{c}$. For energies $\varepsilon \gg E_{c}$ the summation over $\left\{n_{\mu}\right\}$ can be replaced by an integral over $d q_{\mu}$ and

$$
\left\langle[\delta N(\varepsilon)]^{2}\right\rangle=c_{d}\left(\frac{\varepsilon}{E_{c}}\right)^{d / 2}
$$

where $c_{d}$ is a dimensionality-dependent numerical coefficient [4]. The asymptotic results (9.8) and (9.9) have recently been rederived by Argaman, Imry and Smilansky 44 using a more intuitive semiclassical method for electrons in the diffusive regime. The smallenergy universal regime is obtained for times long enough to allow a diffusing electron to ergodically explore all the sample, while for energy intervals larger than the Thouless energy (short times) the correlations depend on the diffusing, unbounded dynamics of the electron, which is dimensionality and disorder dependent. The universal character of the short range eigenvalue correlation and the long range non-universal (dimensionality and disorder dependent) part have been also obtained in numerical simulations of by Dupuis and Montambaux 43] who studied the crossover between the two regimes in an Anderson model.

Given Eq. (9.4), and the above results for the number variance predicted by perturbation theory, we expect the correlation functions of the phase shifts to have a COE-CUE behavior for phase shift separations smaller than

$$
\eta_{c}=\frac{\tau_{W} E_{c}}{\hbar}
$$

Our numerical data summarized in Table I of Appendix A indicates that for the studied samples the critical angle $\eta_{c}$ is smaller than the mean phase shift spacing $\left(r_{c}=\eta_{c} /(2 \pi / M)=\right.$ $\langle g\rangle / 2 \pi<1)$. Therefore they are in the transition regime between the two asymptotic limits (9.8) and (9.9).

In Secs. VI and VIII we have studied the number variance of the phase shifts $\sigma^{2}(r)=$ $\Sigma^{2}(r)-\Sigma_{O E}^{2}(r)$. In particular, from Fig. 8 we can see that $\sigma^{2}(r)$ grows almost linearly from $r \approx r_{c}$. To check our prediction, it is interesting to look at the value of the slope. Whithin the perturbation theory of Ref. [4] the difference between the number variance for the real energy levels and the R.M.T variance is obtained by excluding the term $n_{x}=n_{y}=0$ from the sum (9.7). For a two-dimensional sample the slope of the difference evaluated at $\nu_{c}=E_{c} / \Delta$ is given by

$$
\left(\frac{d}{d e}\left\langle[\delta N(e)]^{2}\right\rangle-\Sigma_{O E}^{2}(e)\right)=\frac{2}{\pi^{2}} \frac{\Delta}{E_{c}} \sum_{\left\{n_{x}, n_{y}\right\} \neq\{0,0\}} \frac{1}{1+\pi^{4}\left(n_{x}^{2}+n_{y}^{2}\right)^{2}} \approx 0.0214 \frac{1}{e_{c}} .
$$

For a quasi-one-dimensional sample the sum is over $n_{x} \neq 0$ and the slope is approximately $0.0045 / e_{c}$. Given the relationship between the statistics of phase shifts and energy levels, we expect that the slope $\phi_{c}=\left.\frac{d}{d r} \sigma^{2}(r)\right|_{r=r_{c}}$ also scales linearly with $1 / r_{c}$. In Fig. 9 we show the value $\phi_{c}$ versus the inverse Thouless energy $1 / r_{c}$. The linear relationship predicted by Eq. (9.11) turns out to be approximately valid. The slope obtained for the two-dimensional case (squares geometries in Fig. 9) agrees within 50\% with the coefficient of (9.11) and is a factor 
of 4 larger than the slope obtained for quasi-one dimensional geometries (roughly the same ratio than for the eigenenergies). Obviously, in those samples, the quasi-one-dimensional limit is not achieved and the approach to this limit depends on the aspect ratio of the sample.

\section{CONCLUSIONS}

In this work we have considered scattering in disordered systems. We calculated the invariant measure of $S$ in the polar decomposition and compared the COE-CUE distributions with those of the global and local approaches to the transfer matrix of disordered systems. We then turned to the study of the scattering phase shifts for different geometries and degree of disorder. For quasi-one dimensional samples in the metallic regime the density and the two-point correlation functions are close to those of the circular ensembles. Increasing the lenght with fixed disorder or the disorder with fixed geometry, we break $S$ into two uncorrelated reflection submatrices. Deep in the quasi- $1 d$ localized regime the decoupling is almost complete and the two-point correlation function of the phase shifts is that of a superposition of two independent circular ensembles (COE or CUE).

Outside quasi-one dimension, the phase shift density differs from the uniform distribution characteristic of Dyson ensembles. This anisotropy comes from short-time processes which yield a non vanishing ensemble average of $S$, and is strongly enhanced in the vicinity of a energy threshold where a new conduction channel appears. Using diagrammatic perturbation theory, we calculated the average value of the reflection amplitudes, finding good agreement with the numerical simulations and establishing the conditions for having the uniform phase shift distribution of the circular ensembles. One needs both energy far from a threshold, weak-disorder, small transverse length and large number of propagating modes. When this is not the case, anisotropy is important and we have to unfold the spectrum in order to study its correlations.

For large transverse lenghts the electron diffusion in the perpendicular direction gives rise to small- $k$ corrections of the two-level from factor $b(k)$ of the [unfolded] phase shift spectrum. This non universal behavior is related to the large-energy (non-ergodic) behavior found by Altshuler and Shklovskil for the energy-level statistics of small metallic particles. This lead us to relate under certain assumptions the scattering phase shift and the energy-level form factors. We numerically checked this relationship for the number variance (fluctuation of the number of states within a given interval). We verified that the non universal discrepancies become increasingly important as the Thouless energy decreases, in a geometry dependent fashion.

One of the most important problems remaining in the random matrix theory of electron transport in disorder systems is the extension of the standard approach outside the quasione-dimensional case. We have shown in this work that anisotropy is an essential ingredient in higher dimensions. The precise form of the decoupling of the $S$ matrix into independent reflections blocks in the one-dimensional localized regime is another interesting problem left for futures studies. Given the recent progress in the theory of parametric correlations [44 [4], the energy dependence of the scattering phase shifts desserves further studies (e. g. the correlations of the characteristic times $\tau_{m}(E)$ (Eq. (B4), slopes in Fig. 10) which we are 
presently developing.

\section{ACKNOWLEDGEMENTS}

We acknowledge helpful discussions with B. Altshuler, H. Baranger, C. Beenakker, E. Bogomolny, S. Feng, P. Leboeuf, P. Mello, G. Montambaux, K. Slevin, U. Smilansky and H. Weidenmuller. Both authors thank the Wissenschaftskolleg zu Berlin for hospitality and fruitful discussions with the participants of the program on Dynamical Systems. This work was partially supported by EEC, Contract No SCC-CT 90-0020.

\section{APPENDIX A: SUMMARY OF THE NUMERICAL SIMULATIONS}

In this appendix we summarize the results of the numerical simulations and we briefly indicate the way they were obtained. The simulations were performed in a tight-binding model with a number of sites $L_{x} \times L_{y}$ and a random on-site disorder of amplitude $W$ by using a recursive Geen function method [47,48. In a magnetic field we use the Peierls substitution to relate the hopping matrix element to the vector potential. The magnetic field is taken to be linearly increasing in the leads from zero to its full value in the disordered region over a distance of the order of the transverse dimension $L_{y}$. For the small fields that we work with the statistical results are independent on the way the field is introduced.

Runs 1-11 in Table I are in the quasi-one-dimensional limit, while runs 12-18 are for square samples and runs 19 for thin slabs. The time limitation for the numerical simulations is the transverse size and that limits the number of samples $(N S)$ considered for large $L_{y}$. The number of modes $N$ was kept low (14-20 in most of the cases) in order to have small Fermi energy $\left(E_{f}\right)$ and wavevector $(k)$ avoiding lattice effects. The Wigner time $\tau_{W}$ is calculated from Eq. (9.2) and provides, through (9.1), the value of the mean level spacing. Using the free-space two-dimensional density of states to give the Weyl term of our disorder region, the level spacing is $\Delta=m L_{x} L_{y} / 2 \pi \hbar^{2}$, and the column showing $\left(\tau_{W} \Delta / \hbar\right)(N / \pi)$ checks the approximate validity of Eq. (9.1) under the hypothesis that the level spacing does not change by the effect of confinement or the disorder. We obtain in most of the cases an agreement within 5-25\% is quite reasonable given the various approximations involved.

The elastic-mean-free path $l$ calculated in Born approximation [49] using samples of transverse dimension $L_{y}$ and a few lattice sites on the longitudinal dimension. In the metallic regime it agrees very well with the mean free path obtained from the Drude formula for the dimensionless conductance $\langle g\rangle=N l \pi / 2 L_{x}$. The Thouless energy in Anderson units is given by $E_{c}=l / L_{x}^{2} \sin k$. The reduced critical angle $r_{c}$ is obtained by scaling the critical angle

$\eta_{c}=\tau_{W} E_{c} / \hbar$ (Eq. (9.10) ) with the mean level spacing $2 \pi / M$. The factor $\phi_{c}$ measuring the discrepancy with the standard ensembles is obtained from Fig. 8 by taking the slope of $\sigma^{2}(r)$ between $r=0.7$ and $r=7$. This is not completely equivalent to the slope at $r_{c}$ since $r_{c}$ and the range of linear behavior of $\sigma^{2}$ varies from sample to sample, but has the advantage of providing a consistent definition for all the cases.

Conductances for individual samples are obtained from Landauer's formula, Eq. (1.2), and then averaged over impurity realizations yielding the average $\langle g\rangle$ and the variance $\left\langle\delta g^{2}\right\rangle$. According to the Thouless formula $\langle g\rangle=2 \pi E_{c} / \Delta$ in the metallic regime, and by using 
the approximate $\Delta$ of the perfect square we obtain an agreement of $25 \%$ with Landauer's

formula. It is important to notice that $r_{c}$ is obtained from $l$ and $\tau_{W}$, directly extracted from the simulations (without any assumption over the form of the conductance). In the localized regime (runs 4,5 and 10) the average conductance and its variance are dominated by special configurations with anomalously large values. Therefore averaging over log is the meaningfull [30] thing to do. In Table I we give as $\langle g\rangle$ for these runs the typical conductance $\exp \langle\log g\rangle$ and $\left\langle\delta(\log g)^{2}\right\rangle$ in the column of the variances.

In the metallic regime the difference between the average conductance without and with magnetic field is the weak locatization correction. Mello and Stone [50] have calculated this correction in the quasi-1d limit by diagrammatic and random matrix approaches and found a value of $1 / 3$, which is somehow larger than the one obtained from Table I. It is rather difficult to reproduce the theoretical value in numerical simulations on disordered strips since getting to the regime of validity of the theory (diffusive, quasi-1D, metallic and magnetic field large enough to kill the time reversal symmetry but small enough for not to alter the classical electron paths) requires extremely large sample sizes [51]. The values of the conductance fluctuations $\left\langle\delta g^{2}\right\rangle$ for zero magnetic field in the metallic regime are in good agreement with the diagrammatic values [2.3] and previous numerical simulations [1, [2. 2 . The halfing of the variance by the effect of the magnetic field is not quite obtained for $\phi / \phi_{0}=2$, but for ratios of the order of 5-10 the factor of 2 it is always found. This is somehow surprising since for $\phi / \phi_{0}=2$ we have already a complete transition towards the unitary ensemble (see Fig. 2).

The mean values of $|\langle\operatorname{Tr} S\rangle|$ are in qualitative agreement with the perturbation result of Eq. (7.9), increasing with disorder and decreasing with the number of modes $N$.

\section{APPENDIX B: RELATION BETWEEN THE SCATTERING PHASE SHIFT AND ENERGY LEVEL STATISTICS}

In this appendix we present, following Bogomolny [40] and Doron and Smilansky [41, a relation between the statistical correlations of the phase shifts and the energy levels in a form appropriate to the scattering which we consider.

Close to the entrance cross sections $\left(x \leq 0\right.$ and $\left.x \geq L_{x}\right)$ the scattering wave functions are given by the asymptotic expressions (2.1) plus the contribution of the evanescent modes:

$$
\begin{gathered}
\Psi_{I}(x, y)=\sum_{n=1}^{N} \frac{1}{k_{n}^{1 / 2}}\left(A_{n} e^{i k_{n} x}+B_{n} e^{-i k_{n} x}\right) \phi_{n}(y)+\sum_{n=N+1}^{\infty} \frac{1}{\left|k_{n}\right|^{1 / 2}} F_{n} e^{\left|k_{n}\right| x} \phi_{n}(y), \quad \text { (B1a) } \\
\Psi_{I I}(x, y)=\sum_{n=1}^{N} \frac{1}{k_{n}^{1 / 2}}\left(C_{n} e^{i k_{n}\left(x-L_{x}\right)}+D_{n} e^{-i k_{n}\left(x-L_{x}\right)}\right) \phi_{n}(y)+\sum_{n=N+1}^{\infty} \frac{1}{\left|k_{n}\right|^{1 / 2}} G_{n} e^{-\left|k_{n}\right|\left(x-L_{x}\right)} \phi_{n}(y) .
\end{gathered}
$$

In Ref. [41], the contribution of the evanescent modes for the evaluation of $\Psi_{I}(x=0, y)$ and $\Psi_{I I}\left(x=L_{x}, y\right)$ is neglected in the semiclassical limit. This sharp cut off at $N+1$ in the sum over $n$ is clearly not exact in the case of our numerical simulations, where $N$ is typically 14 and higher transverse harmonics are needed in order to represent the 
scattering wavefunction at the entrance. Neglecting the evanescent channels, the scattering wave function vanishes at the boundaries $\left(x=0\right.$ and $\left.x=L_{x}\right)$ if $B=-A$ and $C=-D$, implying that the scattering wave function coincides inside the sample with an eigenfunction of the hamiltonian (assuming hard-wall boundary conditions). Therefore, an energy $E$ is an eigenvalue of the closed system whenever $S(E)$ has an eigenvalue -1 [41]

$$
\operatorname{det}(I+S(E))=0
$$

As illustrated in Fig. 10, the energy levels can be obtained by the intersection of the curves $\theta_{m}(E)$ with horizontal lines at $\theta=(2 n+1) \pi$. The accuracy of this quantization condition has been recently tested numerically [53] in the Sinai billiard. Away from the mode thresholds, the errors in the determination of the energy levels are of the order of only a few percent, and they are greatly reduced when a few closed channels are kept. On the other hand, we are not interested in the precise energy levels but in the relationships between their statistics and that of the phase shifts. Towards this end, we have calculated the nearest neighbour distribution of the energy levels determined from (B2) and found, in the metallic regime, good agreement with the corresponding Wigner distributions. From the above quantization condition (B2) the level density can be expressed as

$$
\mathrm{d}(E)=\sum_{n}\left\langle\delta\left(E-E_{n}\right)\right\rangle=\frac{1}{2 \pi \hbar} \sum_{m=1}^{M} \sum_{n=-\infty}^{\infty}(-1)^{n}\left\langle\tau_{m}(E) \exp \left(i n \theta_{m}(E)\right)\right\rangle
$$

where the time associated with the $m^{\text {th }}$ phase shift is

$$
\tau_{m}(E)=\hbar \frac{d \theta_{m}(E)}{d E}
$$

Ignoring correlations between $\theta_{m}$ and $\tau_{m}$ and in the hypothesis that the phase shift distribution is uniform $\left(\left\langle\operatorname{Tr} S^{n}\right\rangle=0\right.$ for all $\left.n \neq 0\right)$ we have Eq. (9.1) of the text

$$
\mathrm{d}(E)=\frac{M}{2 \pi \hbar}\left\langle\tau_{W}(E)\right\rangle
$$

where the Wigner time $\tau_{W}(E)$ is given by $(9.2)$ or

$$
\tau_{W}(E)=\frac{1}{M} \sum_{m=1}^{M} \tau_{m}(E)
$$

${ }^{5}$ If we use the convention $\hat{S}=\left(\begin{array}{cc}t^{\prime} & r \\ r & t\end{array}\right)$ and we ignore the evanescent modes, the condition to have an eigenenergy of the closed system with periodic boundary conditions is that $\hat{S}$ has the eigenvalue 1 , that is, $\operatorname{det}(I-\hat{S}(E))=0$. It is easy to see that $\hat{S}=S U$, with $U=\left(\begin{array}{ll}0 & I \\ I & 0\end{array}\right)$. Therefore, for Dyson Circular Ensembles where the probability distribution is given by the invariant measure in the appropriate space, we do not expect a difference between the statistical properties of $S$ and $\hat{S}$. 
The Wigner time is the mean slope of the curves $\theta_{m}(E)$ in Fig. 10 and has the physical interpretation of the mean time spent by the particles in the scattering region, as can be easily seen from our semiclassical analysis of the transmission amplitude (Appendix C). Notice that the Wigner time resulting from our convention for the $S$ matrix is not the usual delay time but and absolute (always positive) time. In particular, for a perfect (non-disordered) sample it is not zero, but given by $\tau_{W}=2 \hbar / M \sum_{n=1}^{N} d\left(k_{n} L_{x}\right) / d E=\frac{1}{N} \sum_{n=1}^{N} L_{x} / v_{n}$, that is, the average over the propagating modes of the ballistic traversal times.

From its definition (9.3) and the relationship (B3) we can write the density-density correlation function of the energy levels as

$$
\begin{array}{r}
K_{2}(E, E+\varepsilon)=\frac{1}{(2 \pi \hbar)^{2}} \sum_{m, m^{\prime}=1}^{M} \sum_{n, n^{\prime}=-\infty}^{\infty}(-1)^{n+n^{\prime}} \\
\left\langle\tau_{m}(E) \tau_{m^{\prime}}(E) \exp \left(i\left(\theta_{m}(E) n-\theta_{m^{\prime}}(E) n^{\prime}-\tau_{m}(E) n^{\prime} \varepsilon / \hbar\right)\right\rangle-\mathrm{d}(E) \mathrm{d}(E+\varepsilon),\right.
\end{array}
$$

where we have used $\theta_{m^{\prime}}(E+\varepsilon) \approx \theta_{m^{\prime}}(E)+\tau_{m^{\prime}}(E) \varepsilon / \hbar$ for $\varepsilon \ll E$ and we assumed that the $\tau_{m}(E)$ are smooth functions (in the scale of $\varepsilon$ ) which can be taken outside the ensemble average. If the phase shifts are uniformely distributed only the terms with $n=n^{\prime}$ survive the ensemble average and 40,41

$$
K_{2}(\varepsilon)=\mathrm{d}(E) \delta(\varepsilon)+\mathrm{d}^{2}(E)\left(\frac{2 \pi}{M(M-1)} R_{2}\left(\frac{2 \pi}{M} \varepsilon \mathrm{d}(E)\right)-1\right) .
$$

Scaling the energy difference with the mean level spacing $(e=\varepsilon \mathrm{d}(E))$, in the large $M$ limit we obtain (9.4) of the text implying that the phase shifts and the energy levels have the same two-point correlation functions when expressed in terms of their respective reduced variables $(r$ and $e$ ). Notice that the relationship between the phase shift correlations (given by $R_{2}(r)$ or $Y_{2}(r)$ ) and the energy level correlations (given by $K_{2}(e)$ ) is obtained in two steps: first the quantization criterion (B2) (which neglects the evanescent modes) and second assumptions on the homogeneity of the phase shift distribution and the smooth character of $\tau_{m}(E)$.

\section{APPENDIX C: SEMICLASSICAL APPROACH}

In this appendix we present a semiclassical treatment of the scattering in a wave-guide geometry with disorder, for understanding of the non-zero average of the diagonal reflection amplitudes and of the various time scales in the problem. The semiclassical approximation to the transmission and reflection amplitudes can be obtained by replacing the Green functions $G_{k}$ of Eqs. (7.3) by their semiclassical path-integral expression [54]

$$
G_{k}^{\mathrm{scl}}\left(y^{\prime}, y\right)=\frac{2 \pi}{(2 \pi i \hbar)^{3 / 2}} \sum_{s\left(y, y^{\prime}\right)} \sqrt{D_{s}} \exp \left(\frac{i}{\hbar} S_{s}\left(y^{\prime}, y, E_{f}\right)-i \frac{\pi}{2} \mu_{s}\right) .
$$

The sum is over classical trajectories $s$ between the arguments of the Green function (we will be omiting the $x$ dependence since it will be assumed $x=0$ or $L_{x}$ depending whether we are considering reflection or transmission), $S_{s}$ is the action integral along the path $s$ at 
energy $E_{f}=\hbar^{2} k^{2} / 2 m=m v^{2} / 2$, the stability prefactor is $D_{s}=\left(v\left|\cos \theta^{\prime}\right| / m\right)^{-1}\left|\left(\partial \theta / \partial y^{\prime}\right)_{y}\right|$, $\theta$ and $\theta^{\prime}$ are the incoming and outgoing angles, and $\mu$ is the Maslov index given by the number of constant-energy conjugate points [54]. Performing the transverse integrations by stationary-phase approximation, valid in the $\hbar \rightarrow 0$ limit applicable to the many-channel case that we are interested in, we obtain 55

$$
r_{b a}^{\mathrm{scl}}=-\frac{\sqrt{2 \pi i \hbar}}{2 L_{y}} \sum_{s(\bar{a}, \bar{b})} \operatorname{sgn}(\bar{a}) \operatorname{sgn}(\bar{b}) \sqrt{\tilde{D}_{s}} \exp \left(\frac{i}{\hbar} \tilde{S}_{s}\left(\bar{b}, \bar{a}, E_{f}\right)-i \frac{\pi}{2} \tilde{\mu}_{s}\right)
$$

where the sum is taken over trajectories $s$ between the cross section at $x=0$ with incoming and outgoing angles $\theta$ and $\theta^{\prime}$ such that $\sin \theta=\bar{a} \pi / k L_{y}$ and $\sin \theta^{\prime}=\bar{b} \pi / k L_{y}(\bar{a}= \pm a$, $\bar{b}= \pm b)$. Since $\bar{a}$ and $\bar{b}$ are integers we see that the semiclassical approximation yields the intuitive result that only trajectories which enter and exit at discrete angles corresponding to the allowed quantized transverse momenta contribute to reflection. The reduced action (virial) is

$$
\tilde{S}\left(\bar{b}, \bar{a}, E_{f}\right)=S\left(y_{0}^{\prime}, y_{0}, E_{f}\right)+\hbar \pi \bar{a} y_{0} / L_{y}-\hbar \pi \bar{b} y_{0}^{\prime} / L_{y}
$$

the new pre-exponential factor is

$$
\tilde{D}_{s}=\frac{1}{m v\left|\cos \theta^{\prime}\right|}\left|\left(\frac{\partial y}{\partial \theta^{\prime}}\right)_{\theta}\right|
$$

and the Maslov index $\tilde{\mu}$ is given by $\mu$ and the signs of the second derivatives of the action (see Ref. [55]). A similar expression holds for the transmission amplitudes, but the trajectories are now taken from the cross-section at $x=0$ to that at $x=L_{x}$. The Kronecker $\delta_{a, b}$ of the reflection amplitude, Eq. (7.3b), has been taken care off by trajectories going from $y$ to $y^{\prime}$ along the cross section $x=0$ (without entering the disordered region) not included in Eq. (C2) [55]. The rapid increase of the mean value of the reflection amplitude for high modes can be traced to the $\cos \theta^{\prime}$ of Eq. (C4) since $\theta^{\prime}$ approaches $\pi / 2$ as $a$ increases towards $N$.

When taking the average of the matrix elements of $S$ over different impurity configurations, the long trajectories will have a rapidly varying phase which leads to zero average, while the short reflection trajectories which do not explore the bulk of the sample will survive the average. In particular, trajectories corresponding to high mode numbers will have a large injection angle which makes very likely their reflection within a mean free path $l$ from the entrance. The absence of selfaveraging to zero for the diagonal reflection elements subsists in the limit of $L_{x} \rightarrow \infty$ of a semi-infinite strip, since it is a consequence of fast (short trajectory) processes. These short processes are the only ones contributing to $\langle\operatorname{Tr} S\rangle$ in the large $N$-limit and this is the reason why in the numerics and in Eq. (7.9) the trace of the scattering matrix depends only on the properties at the entrance of the sample. However, the reflected trajectories have a wide distribution of lengths, and we do not have as in Nuclear Physics [56] a clear-cut separation between direct (or fast) and long processes.

In order to put these ideas in a more quantitative level, we calculate the semiclassical approximation to $\operatorname{Tr} S^{n}$. For $n=1$ we only need the trace of the reflection matrices $r$ and $r^{\prime}$, which can be evaluated with the aid of Poisson summation formula, 


$$
\begin{array}{r}
\operatorname{Tr} r^{\mathrm{scl}=} \sum_{a=1}^{N} r_{a a}=-\frac{\sqrt{2 \pi i \hbar}}{2 L_{y}} \sum_{m=-\infty}^{\infty} \int_{1 / 2}^{N+1 / 2} d u \exp (2 \pi i m u) \sum_{s(\bar{u}, \overline{\bar{u}})} \operatorname{sgn}(\bar{u}) \operatorname{sgn}(\overline{\bar{u}}) \\
\sqrt{\tilde{D}_{s}} \exp \left(\frac{i}{\hbar} \tilde{S}_{s}\left(\overline{\bar{u}}, \bar{u}, E_{f}\right)-i \frac{\pi}{2} \tilde{\mu}_{s}\right)
\end{array}
$$

where $\bar{u}, \overline{\bar{u}}= \pm u$, not necessarily an integer. The stationary-phase condition for the integral over $u$ is

$$
\operatorname{sgn}(\overline{\bar{u}}) y_{0}^{\prime}-\operatorname{sgn}(\bar{u}) y_{0}=2 m L_{y}
$$

Since $0<y_{0}, y_{0}^{\prime}<L_{y}$ the only solution is $\overline{\bar{u}}=\bar{u}, y_{0}^{\prime}=y_{0}$, and $m=0$, i.e., the trajectory closes itself at the entrance cross-section with a specular reflection, where the transverse momentum (in the $y$ direction) is conserved. Keeping only the $m=0$ term corresponds to the approximation of replacing the sum over $a$ in $\operatorname{Tr} r^{\text {scl }}$ by an integral; the stationary-phase argument indicates that this is indeed the leading term in $\hbar$. We then have

$$
\operatorname{Tr} r^{\mathrm{scl}}=-\frac{i \pi \hbar}{\sqrt{2} L_{y}} \sum_{s\left(y_{0}, \bar{u}_{0} ; y_{0}, \bar{u}_{0}\right)} \sqrt{\hat{D}_{s}} \exp \left(\frac{i}{\hbar} S_{s}\left(y_{0}, y_{0}, E_{f}\right)-i \frac{\pi}{2} \hat{\mu}_{s}\right),
$$

$S_{s}$ is the action integral along the classical path $s$, the stability prefactor is

$$
\hat{D}_{s}=\tilde{D}_{s}\left|\left(\frac{\partial^{2} \tilde{S}_{s}}{\partial a^{2}}\right)_{b}+\left(\frac{\partial^{2} \tilde{S}_{s}}{\partial b^{2}}\right)_{a}\right|_{\bar{a}=\bar{b}=\bar{u}_{0}}^{-1},
$$

and the new Maslov index $\hat{\mu}_{s}$ is given by $\tilde{\mu}_{s}$ and the sign of the second derivative of the reduced action. For the semiclassical approximation to $\operatorname{Tr} r^{\prime}$ we have a similar expression, given in terms of trajectories who have a specular reflection at the $x=L_{x}$ cross-section.

Higher powers of $S$ involve products of the matrices $r, t, r^{\prime}, t^{\prime}$. For instance, the reflection submatrix of $S^{2}$ is $r r+t^{\prime} t$, and represents the two possible ways of returning to the entrance cross-section touching only once one of the vertical boundaries. The matrix products of the reflection and transmission amplitudes can also be obtained with the aid of Poisson summation formula, resulting in a stationary-phase condition very similar to Eq. (C6) (but now the terms with $y_{0}$ and $y_{0}^{\prime}$ come from actions of different trajectories, i.e. one from $t$ and the other from $t^{\prime}$ ) which indicates that we also have specular reflections at each bounce with the vertical walls. Once we take the trace we arrive at the intuitive result that $\operatorname{Tr} S^{n}$ is given in a semiclassical approximation by closed trajectories, of a system with hard walls at the extreme cross-sections, with $n$ bounces with the vertical walls.

Blümel and Smilansky [19 expressed $\operatorname{Tr} S^{n}$ semiclassically in terms of periodic orbits of the Poincaré scattering map, while in Ref. [41] it was shown that a periodic orbit of order $n$ of the mapping corresponds to a periodic orbit of the closed system which hits $n$ times the extreme cross-sections. This is precisely our statement of last paragraph.

The semiclassical approximation to $\operatorname{Tr} S^{n}$, given by closed orbits hitting $n$ times the vertical hard walls, is very useful since we can identify high values of $n$ with long trajectories. The lower harmonics of $\rho(\theta)$ are given by the ensemble average over short orbits, which is not zero in general; while the higher harmonics are given by long orbits leading to self averaging. 
This explains why $\rho(\theta)$ is relatively smooth and justifies the usefulness of our diagrammatic approach yielding only $\rho_{1}(\theta)$.

According to Eq. (5.7) the two-level form factor is given by the ensemble average of $\left|\operatorname{Tr} S^{n}\right|^{2}$, therefore the semiclassical approach also allows us to understand the phase shift correlations. The difference in the semiclassical approaches to the mean density and the TLFF is that the former is expressed as a one-phase sum while the latter a two-phase sum given by products of two periodic orbits of order $n$. In a diagonal approximation we would take both trajectories to be the same or symmetrically related. This yields a factor of two difference between the cases with and without magnetic field, which we obtain for small values of $n$ (or in the slope at the origen) in our numerical simulations (see Fig. 2). Since the two curves for the TLFF must have the same area, they have to cross at one point, indicating that the diagonal approximation ceases to be valid for long trajectories. Therefore, for relatively low $n$ we can approximate the Fourier components $s_{n}^{M}$ as simply given by periodic orbits hitting $n$ times the vertical cross sections.

The semiclassical approach allows us to give an intuitive interpretation of the Wigner time. According to Eqs. (9.2) and (C2) we have

$$
\tau_{W}(E)=\frac{\hbar}{i M} \sum_{i, j}^{M} S_{j i}^{*} \frac{d S_{j i}}{d E}=\frac{\hbar}{i N} \sum_{a, b}^{M} \sum_{s(\bar{a}, \bar{b})} \sum_{s^{\prime}(\bar{a}, \bar{b})} \sqrt{\tilde{D}_{s} \tilde{D}_{s^{\prime}}} T_{s^{\prime}} \exp \left(\frac{i}{\hbar}\left(\tilde{S}_{s}-\tilde{S}_{s^{\prime}}\right)-i \frac{\pi}{2}\left(\tilde{\mu}_{s}-\tilde{\mu}_{s^{\prime}}\right)\right) .
$$

The sum is over all pairs of trajectories $s$ and $s^{\prime}$ contributing to $r, r^{\prime}, t$ or $t^{\prime}$. In performing the ensemble average the short trajectories (where the diagonal approximation is valid) will dominate and for $s=s^{\prime}$ we have

$$
\left\langle\tau_{W}(E)\right\rangle=\frac{\hbar}{M} \sum_{a b}^{M} \sum_{s(\bar{a}, \bar{b})} \tilde{D}_{s} T_{s},
$$

giving the standard interpretation of the Wigner time as the mean scattering time (mean time of the classical scattering trajectories averaged with their stability prefactor) or the inverse escape rate [57] from the disordered region.

The criterion developed Sec. $\mathbb{X}$ ] was to measure the departures from the circular ensembles by the parameter $\eta_{c}=\tau_{W} E_{c} / \hbar$, which is simply the ratio between the scattering and the diffusion times across the sample. The agreement with RMT obtained for large values of $\eta_{c}$ translates into a Wigner time much larger than the diffusing time for traversing the structure $\left(L_{x}^{2} / D\right)$. That is, the particle spends in the disordered region enough time to sample it before leaving.

Separating the reflection and transmission trajectories $s$ of Eq. (C10) we see that, at the semiclassical level, the Wigner time can be decomposed into transmission and reflection components. At the quantum mechanical level, this is not possible since the matrices $r, r^{\prime}, t$ and $t^{\prime}$ are not unitary, and for a given impurity realization we obtain an imaginary part when using these matrices instead of $S$ in Eq. (9.2). However, these imaginary parts vanish under ensemble average giving well-defined transmission and reflection Wigner times. These partial times can be related with the classical traversal times of a diffusion through a disordered region connected with leads (diffusion from an entrance to an exit [58.59]). Calling $\tau_{e}=l / v_{f}$ 
the elastic scattering time, the quasi-one-dimensional case can be discretized as a diffusion in a one-dimensional lattice with $m=L_{x} / l$ sites. In this model it has been show 58,59 that the mean traversal and reflection times are given by

$$
\begin{gathered}
\tau_{T}=\tau_{e} m\left(\frac{1}{3} m+\frac{2}{3}\right) \\
\tau_{R}=\tau_{e}\left(\frac{2}{3} m+\frac{1}{3}\right) .
\end{gathered}
$$

Therefore, in the large $m$-limit, the mean traversal time is reduced by a factor of three with respect to the diffusion time in an infinite chain, $\tau_{T} \approx 1 / 3 \tau_{e} m^{2}=1 / 3\left(L_{x}^{2} / 2 D\right)$. The classical transmission and reflection probabilities are

$$
\begin{aligned}
& T=\frac{1}{m+1}, \\
& R=\frac{m}{m+1},
\end{aligned}
$$

and the total dwell time is

$$
\tau_{D}=\tau_{T} T+\tau_{R} R=\tau_{e} m=L_{x} / v_{f},
$$

consistent with our findings that the Wigner time is approximately independent of disorder and coincides with the ballistic traversal time. For quasi-one-dimensional geometries the partial Wigner times obtained in the simulations after impurity average are in relatively good agreement with the average diffusion times $\tau_{T} T$ and $\tau_{R} R$. From Eqs. (C11) and (C12) we can also calculate the mean lenght of trajectories contributing to $s_{n}^{M}$, that is, those hitting $n$ times the vertical walls at the entrance of the disordered region. For instance, for $n=2$ we have

$$
\tau_{2}=\frac{2 \tau_{T} T^{2}+2 \tau_{R} R^{2}}{T^{2}+R^{2}}
$$

In the large $m$-limit, trajectories with $n$ bounces will have a typical length proportional to $n$.

\section{APPENDIX D: INVARIANT MEASURE OF S IN THE POLAR DECOMPOSITION WITHOUT TIME-REVERSAL SYMMETRY}

In this appendix we find the metric of the scattering matrix in the polar decomposition for the case where there is no time-reversal symmetry. The calculation follows the same lines of that in Sec. III, the difference coming from the fact that instead of the decomposition (3.1) we now have

$$
S=X^{\dagger} \Sigma X=U^{\prime} \Gamma U=Y^{\prime} Y
$$


The unitary matrix $X$ diagonalizes $S, U$ and $U^{\prime}$ are independent block-diagonal unitary matrices given by the polar decomposition (2.9). $Y$ and $Y^{\prime}$ are independent unitary matrices that allow to define the metric of $S$. Diagonalizing $\Gamma$ as in Eq. (3.4) we have the same $Y$ as before (Eq. (3.8)) and

$$
Y^{\prime}=U^{\prime} O^{\mathrm{T}} F=\left(\begin{array}{lr}
u^{(3)} \mathcal{P} & i u^{(3)} \mathcal{Q} \\
u^{(4)} \mathcal{Q} & -i u^{(4)} \mathcal{P}
\end{array}\right)
$$

The infinitesimal variations of $Y, u^{(1)}$, and $u^{(2)}$ are given by

(3.9) and (3.10) in terms of the antihermitian matrices $\delta Y, \delta u^{(1)}$ and $\delta u^{(2)}$. Analogously, we can write

$$
\begin{gathered}
d Y=Y^{\prime} \delta Y^{\prime}, \\
d u^{(l)}=u^{(l)} \delta u^{(l)}, \quad l u^{(l)}=d a^{(l)}+i d s^{(l)}, \quad l=3,4 .
\end{gathered}
$$

Therefore the infinitesimal variations of $S$ are given by

$$
d S=Y^{\prime}\left(\delta Y^{\prime}+\delta Y\right) Y=Y^{\prime}(i d \tilde{H}) Y .
$$

The hermitian matrix $d \tilde{H}$ defines the metric of $S$ by [7]

$$
\mu(d S)=\prod_{i \leq j}^{2 N} \operatorname{Re}(d \tilde{H})_{i j} \prod_{i<j}^{2 N} \operatorname{Im}(d \tilde{H})_{i j}
$$

and is given by

$$
\begin{aligned}
& d \tilde{H}=2\left(\begin{array}{cc}
0 & \mathcal{P} d \mathcal{Q}-\mathcal{Q} d \mathcal{P} \\
\mathcal{P} d \mathcal{Q}-\mathcal{Q} d \mathcal{P} & 0
\end{array}\right)+ \\
& +\left(\begin{array}{lr}
\mathcal{P}\left(d s^{(1)}+d s^{(3)}\right) \mathcal{P}+\mathcal{Q}\left(d s^{(2)}+d s^{(4)}\right) \mathcal{Q} & -\mathcal{P}\left(d a^{(1)}-d a^{(3)}\right) \mathcal{Q}+\mathcal{Q}\left(d a^{(2)}-d a^{(4)}\right) \mathcal{P} \\
\mathcal{Q}\left(d a^{(1)}-d a^{(3)}\right) \mathcal{P}-\mathcal{P}\left(d a^{(2)}-d a^{(4)}\right) \mathcal{Q} & \mathcal{Q}\left(d s^{(1)}+d s^{(3)}\right) \mathcal{Q}+\mathcal{P}\left(d s^{(2)}+d s^{(4)}\right) \mathcal{P}
\end{array}\right) \\
& -i\left(\begin{array}{ll}
\mathcal{P}\left(d a^{(1)}+d a^{(3)}\right) \mathcal{P}+\mathcal{Q}\left(d a^{(2)}+d a^{(4)}\right) \mathcal{Q} & \mathcal{P}\left(d s^{(1)}-d s^{(3)}\right) \mathcal{Q}-\mathcal{Q}\left(d s^{(2)}-d s^{(4)}\right) \mathcal{P} \\
-\mathcal{Q}\left(d s^{(1)}-d s^{(3)}\right) \mathcal{P}+\mathcal{P}\left(d s^{(2)}-d s^{(4)}\right) \mathcal{Q} & \mathcal{Q}\left(d a^{(1)}+d a^{(3)}\right) \mathcal{Q}+\mathcal{P}\left(d a^{(2)}+d a^{(4)}\right) \mathcal{P}
\end{array}\right)
\end{aligned}
$$

In order to calculate the invariant measure of $S$ it is convenient to break the product (D6) over the real and imaginary parts of the elements of $d \tilde{H}$ as

$$
\begin{gathered}
\prod_{a=1}^{N} \operatorname{Re}(d \tilde{H})_{a, a+N}=\prod_{a=1}^{N} \frac{1}{2 \sqrt{\lambda_{a}}\left(1+\lambda_{a}\right)} d \lambda_{a}, \\
\prod_{a<b}^{N} \operatorname{Im}(d \tilde{H})_{a, b} \operatorname{Im}(d \tilde{H})_{a+N, b+N} \operatorname{Re}(d \tilde{H})_{a, b+N} \operatorname{Re}(d \tilde{H})_{b, a+N}=\prod_{a<b}^{N}\left(\frac{\lambda_{a}}{\lambda_{a}+1}-\frac{\lambda_{b}}{\lambda_{b}+1}\right) \prod_{l=1}^{4} d a_{a b}^{(l)},
\end{gathered}
$$




$$
\prod_{a<b}^{N} \operatorname{Re}(d \tilde{H})_{a, b} \operatorname{Re}(d \tilde{H})_{a+N, b+N} \operatorname{Im}(d \tilde{H})_{a, b+N} \operatorname{Im}(d \tilde{H})_{b, a+N}=\prod_{a<b}^{N}\left(\frac{\lambda_{a}}{\lambda_{a}+1}-\frac{\lambda_{b}}{\lambda_{b}+1}\right) \prod_{l=1}^{4} d s_{a b}^{(l)}
$$

$$
\begin{gathered}
\prod_{a=1}^{N} \operatorname{Re}(d \tilde{H})_{a, a} \operatorname{Re}(d \tilde{H})_{a+N, a+N} \operatorname{Im}(d \tilde{H})_{a, a+N}=\prod_{a=1}^{N}\left(\mathcal{P}_{a}^{2}\left(d s_{a a}^{(1)}+d s_{a a}^{(3)}\right)+\mathcal{Q}_{a}^{2}\left(d s_{a a}^{(2)}+d s_{a a}^{(4)}\right)\right) \times \\
\left(\mathcal{Q}_{a}^{2}\left(d s_{a a}^{(1)}+d s_{a a}^{(3)}\right)+\mathcal{P}_{a}^{2}\left(d s_{a a}^{(2)}+d s_{a a}^{(4)}\right)\right) \times\left(\mathcal{P}_{a} \mathcal{Q}_{a}\left(d s_{a a}^{(1)}-d s_{a a}^{(3)}\right)-\mathcal{Q}_{a} \mathcal{P}_{a}\left(d s_{a a}^{(2)}-d s_{a a}^{(4)}\right)\right)
\end{gathered}
$$

The last product, Eq. (D8d), shows the redundancy of the polar decomposition in the absence of time-reversal symmetry. The matrix $S$ is unchanged if in its polar decomposition we make the transformation [50]

$$
U \rightarrow G U, \quad \quad U^{\prime} \rightarrow U^{\prime} G^{\dagger}, \quad G=\left(\begin{array}{cc}
\mathcal{G} & 0 \\
0 & \mathcal{G}
\end{array}\right),
$$

where the blocks $\mathcal{G}$ are pure-phase diagonal matrices, $\mathcal{G}_{a}=\exp \left(i \eta_{a}\right)$. Under this transformation we have

$$
d s_{a a}^{(l)} \rightarrow d s_{a a}^{(l)} \pm d \eta_{a}, \quad l=1,2(3,4)
$$

and the r.h.s. of Eq. (D8d) remains unchanged. Since $d \eta_{a}$ are arbitrary we take them equal to $d s_{a a}^{(1)}$ [50], and when multiplying both sides of the equation above by $\prod_{a=1}^{N} d s_{a a}^{(1)}$ we obtain the transformation of the volume element as

$$
\prod_{a=1}^{N} \operatorname{Re}(d \tilde{H})_{a, a} \operatorname{Re}(d \tilde{H})_{a+N, a+N} \operatorname{Im}(d \tilde{H})_{a, a+N} d s_{a a}^{(1)}=\prod_{a=1}^{N}\left(\frac{\sqrt{\lambda_{a}}}{\lambda_{a}+1}\right) \prod_{l=1}^{4} d s_{a a}^{(l)} .
$$

As in the time-symmetric case, we express the jacobian of the transformation in the Gibbs form (4.2), with an inverse effective temperature $\beta=2$ and an effective hamiltonian (4.3) given by the same logarithmic interaction as before (Eq. (4.4)) and a one-body potential:

$$
V(\lambda)=N \log (1+\lambda),
$$

which coincides with (4.5) up to order $N$ terms but differs in the terms of order unity $\left(N^{0}\right)$. 


\section{REFERENCES}

[1] For reviews see Mesoscopic Phenomena in Solids (North-Holland, Amsterdam, 1991) ed. by B. L. Altshuler, P. A. Lee, and R. A. Webb.

[2] B. L. Altshuler, Zh. Eksp. Teor. Fiz. 41, 648 (1985) [Sov. Phys. JETP Lett. 41, 6481 (1985)].

[3] P.A.Lee, A.D.Stone and H.Fukuyama, Phys. Rev. B35, 1039 (1987).

[4] B.L. Altshuler and B.I. Shklovskiu, Zh. Eksp. Teor. Fiz. 91, 220 (1986) [Sov. Phys. JETP 64, 127 (1986)].

[5] Y. Imry, Europhys. Lett. 1, 249 (1986).

[6] E. P. Wigner, Ann. Math. 53, 36 (1953).

[7] F. J. Dyson, J. Math. Phys. 3, 140 (1962).

[8] C. E. Porter Statistical Theory of Spectra: Fluctuations (Academic, New York, 1965).

[9] M. L. Mehta Random Matrices and the Statistical Theory of Energy Levels (Academic, New York, 1967).

[10] O. Bohigas, M.J. Giannoni, and C. Schmit, Phys. Rev. Lett. 52, 1 (1984).

[11] O. Bohigas, in Chaos and Quantum Physics (North-Holland, New York, 1991) ed. by M.-J. Giannoni, A. Voros, and J. Zinn-Justin.

[12] L. P. Gorkov and G. M. Eliashberg, Zh. Eksp. Teor. Fiz. 48, 1407 (1965) [Sov. Phys. JETP 21, 940 (1965)].

[13] K. B. Efetov, Adv. Phys. 32, 53 (1983).

[14] K. A. Muttalib, J.-L. Pichard, and A. D. Stone Phys. Rev. Lett. 59, 2475 (1987).

[15] A. D. Stone, P. A. Mello, K. A. Muttalib, and J.-L. Pichard, and in Ref. [1].

[16] F. J. Dyson, J. Math. Phys. 13, 90 (1972).

[17] R. A. Jalabert, J.-L. Pichard, and C. W. J. Beenakker, Europhys. Lett. 27, 255 (1994).

[18] H. U. Baranger and P. A. Mello, Phys. Rev. Lett. 73, 142 (1994).

[19] R. Blümel and U. Smilansky, Phys. Rev. Lett. 64, 241 (1989).

[20] E. Doron and U. Smilansky, Nucl. Phys. A 545, 455c (1992).

[21] P. A. Mello , P. Pereyra, and N. Kumar, Ann. Phys. 181, 290, (1988).

[22] P. A. Mello and J.-L. Pichard, J. Phys. (France) 1, 493, (1991).

[23] K. Frahm, unpublished.

[24] K. Slevin, J.-L. Pichard, and K. A. Muttalib, J. Phys. (France) 3, 1387, (1993).

[25] C. W. J. Beenakker, Phys. Rev. Lett. 70, 1155 (1993); Phys. Rev. B 47, 15763, (1993).

[26] This remarkable fact, which can be shown by a direct evaluation of the conductance fluctuations from the statistics of matrix elements $S_{i j}$ was brought to our attention by P. Mello (Private communication).

[27] O.N. Dorokhov, Solid State Comm. 51, 381 (1984).

[28] P.A. Mello, Phys. Rev. Lett. 60, 1089 (1988).

[29] C. W. J. Beenakker and B. Rejaei, Phys. Rev. Lett. 71, 3689 (1993).

[30] J.-L. Pichard in Quantum Coherence in Mesoscopic Systems ed. by B. Kramer, NATO ASI Series B 254 (Plenum, New York, 1991).

[31] Y. Avishai, J.-L. Pichard, and K. A. Muttalib, J. Phys. (France) 3, 2343, (1993).

[32] A. Pandey, Annals of Phys. (NY) 134, 110 (1981).

[33] F. Borgonovi and I. Guarneri, Phys. Rev. E 48, R2347, (1993).

[34] D. S. Fisher and P. A. Lee, Phys. Rev. B 23, 6851, (1981). 
[35] A. D. Stone in Physics of Nanostructures (IOP, Bristol, 1992) ed. by J. H. Davies and A. R. Long.

[36] E. Akkermans, P. E. Wolf, R. Maynard, and G. Maret, J. Phys. (France) 49, 71, (1988).

[37] S. Iida, H. A. Weidenmüller, and J. A. Zuk Phys. Rev. Lett. 64, 583 (1990); and Annals of Phys. 200, 219 (1990).

[38] P. A. Mello and S. Tomsovic, Phys. Rev. B 46, 15963 (1992).

[39] K. Slevin, J.-L. Pichard, and P. A. Mello, Europhys. Lett. 16, 649 (1991).

[40] E. Bogomolny, unpublished 1991.

[41] E. Doron and U. Smilansky, Phys. Rev. Lett. 68, 1255 (1992); Nonlinearity 5, 1055 (1992).

[42] N. Argaman, Y. Imry, and U. Smilansky, Phys. Rev. B 47, 4440, (1993).

[43] N. Dupuis and G. Montambaux, Phys. Rev. B 43, 14390, (1991).

[44] A. Szafer and B. L. Altshuler, Phys. Rev. Lett. 70, 587 (1993).

[45] B. D. Simons and B. L. Altshuler, Phys. Rev. Lett. 70, 4063 (1993).

[46] C. W. J. Beenakker, Phys. Rev. Lett. 70, 4126 (1993).

[47] P. A. Lee and D. S. Fisher, Phys. Rev. Lett. 47, 882, (1981).

[48] H. U. Baranger, D. P. DiVincenzo, R. A. Jalabert, and A. D. Stone, Phys. Rev. B 44, 10637 (1991).

[49] H. U. Baranger, Phys. Rev. B 42, 11479, (1990).

[50] P. A. Mello and A. D. Stone, Phys. Rev. B 44, 3559, (1991).

[51] H. U. Baranger, private communication.

[52] A. Levy Yeyati, Phys. Rev. B 45, 14189 (1992).

[53] H. Schanz and U. Smilansky, unpublished.

[54] M. C. Gutzwiller, in Chaos in Classical and Quantum Mechanics, (Springer-Verlag, Berlin, 1990); and in Ref. [11].

[55] H. U. Baranger, R. A. Jalabert, and A. D. Stone, Chaos, 3, 665 (1993).

[56] D. Brink and R. Stephen, Phys. Lett. 5, 77, (1963).

[57] E. Doron, U. Smilansky, and A. Frenkel, Physica D 50, 367 (1991).

[58] R. Landauer and M. Büttiker, Phys. Rev. B 36, 6255 (1987).

[59] M. E. Fisher, IBM J. Res. Develop. 32, 76 (1988). 


\section{TABLES}

\begin{tabular}{|c|c|c|c|c|c|c|c|c|c|c|c|c|c|c|c|}
\hline run & $\phi / \phi_{0}$ & $N S$ & $A R$ & $L_{y}$ & $N$ & $E_{f}$ & $k$ & $W$ & $\frac{\tau_{W} \Delta}{\hbar} \frac{N}{\pi}$ & $l$ & $r_{c}$ & $\phi_{c}$ & $\langle g\rangle$ & $\left\langle\delta g^{2}\right\rangle$ & $|\langle\operatorname{Tr} S\rangle|$ \\
\hline R1 & 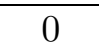 & 5000 & 4 & 34 & 14 & -2.5 & 1.32 & 1 & 1.20 & 28 & 0.67 & $\overline{0.007}$ & 4.14 & 0.13 & 0.25 \\
\hline F1 & 2 & 5000 & & & & & & & & & & & 4.23 & 0.09 & \\
\hline $\mathrm{R} 2$ & 0 & 1400 & 4 & 34 & 14 & -2.5 & 1.32 & 1.5 & 1.24 & 13 & 0.32 & 0.028 & 2.13 & 0.13 & 0.38 \\
\hline $\mathrm{F} 2$ & 2 & 1400 & & & & & & & & & & & 2.23 & 0.08 & \\
\hline R3 & 0 & 700 & 4 & 34 & 14 & -2.5 & 1.32 & 2 & 1.20 & 8.3 & 0.20 & 0.042 & 1.11 & 0.11 & 0.51 \\
\hline F3 & 2 & 700 & & & & & & & & & & & 1.26 & 0.08 & \\
\hline $\mathrm{R} 4$ & 0 & 2500 & 4 & 34 & 14 & -2.5 & 1.32 & 4 & 1.24 & 2.8 & & & 0.02 & 4.98 & 1.68 \\
\hline F4 & 2 & 2500 & & & & & & & & & & & 0.03 & 4.25 & \\
\hline $\mathrm{R} 5$ & 0 & 700 & 4 & 34 & 14 & -2.5 & 1.32 & 6 & 0.34 & 1.8 & & & $10^{-7}$ & 24.9 & 3.60 \\
\hline F5 & 2 & 700 & & & & & & & & & & & $2 \times 10^{-7}$ & 24.8 & \\
\hline R6 & 0 & 700 & 4 & 34 & 20 & -1.36 & 1.90 & 1.5 & 1.60 & 12 & 0.37 & 0.028 & 2.62 & 0.13 & 0.15 \\
\hline $\mathrm{R} 7$ & 0 & 500 & 7 & 34 & 20 & -1.36 & 1.90 & 1 & 1.53 & 27 & 0.37 & 0.025 & 3.47 & 0.13 & 0.13 \\
\hline $\mathrm{R} 8$ & 0 & 1400 & 10 & 34 & 14 & -2.5 & 1.32 & 1 & 1.21 & 28 & 0.26 & 0.033 & 1.91 & 0.13 & 0.20 \\
\hline F8 & 5 & 1400 & & & & & & & & & & & 2.07 & 0.07 & \\
\hline R9 & 0 & 700 & 10 & 34 & 20 & -1.36 & 1.90 & 1 & 1.45 & 27 & 0.30 & 0.028 & 2.50 & 0.13 & 0.19 \\
\hline R10 & 0 & 700 & 30 & 34 & 14 & -2.5 & 1.32 & 1 & 1.27 & 28 & & & 0.45 & 0.58 & 0.28 \\
\hline F10 & 15 & 700 & & & & & & & & & & & 0.69 & 0.22 & \\
\hline R11 & 0 & 600 & 4 & 68 & 14 & -3.56 & 0.68 & 1 & 1.11 & 24 & 0.34 & 0.036 & 1.85 & 0.13 & 1.14 \\
\hline $\mathrm{R} 12$ & 0 & 2500 & 1 & 68 & 14 & -3.56 & 0.68 & 1 & 1.12 & 24 & 1.35 & 0.012 & 5.49 & 0.18 & 1.15 \\
\hline F12 & 2 & 1400 & & & & & & & & & & & 5.55 & 0.15 & \\
\hline $\mathrm{R} 13$ & 0 & 700 & 1 & 68 & 14 & -3.56 & 0.68 & 1.5 & 1.10 & 11 & 0.60 & 0.045 & 3.19 & 0.18 & 2.16 \\
\hline R14 & 0 & 700 & 1 & 68 & 14 & -2.5 & 0.68 & 2 & 0.97 & 5.9 & 0.30 & 0.093 & 1.96 & 0.17 & 3.28 \\
\hline $\mathrm{R} 15$ & 0 & 700 & 1 & 68 & 20 & -3.17 & 0.95 & 1.5 & 1.14 & 13 & 0.98 & 0.021 & 5.22 & 0.18 & 1.48 \\
\hline R16 & 0 & 700 & 1 & 68 & 20 & -3.17 & 0.95 & 2.0 & 1.16 & 7.1 & 0.54 & 0.060 & 3.27 & 0.17 & 2.37 \\
\hline R17 & 0 & 500 & 1 & 68 & 25 & -2.76 & 1.18 & 1.5 & 1.21 & 14 & 1.30 & 0.015 & 6.86 & 0.19 & 1.00 \\
\hline R18 & 0 & 400 & 1 & 68 & 28 & -2.5 & 1.32 & 1.5 & 1.25 & 14 & 1.41 & 0.016 & 7.68 & 0.19 & 0.93 \\
\hline R19 & 0 & 600 & $1 / 4$ & 140 & 14 & -3.90 & 0.33 & 1 & 1.05 & 11 & 0.08 & 0.068 & 6.12 & 0.27 & 4.36 \\
\hline F19 & 2 & 200 & & & & & & & & & & & 6.13 & 0.09 & \\
\hline
\end{tabular}

TABLE I. Runs with zero magnetic field are indicated by $\mathrm{R}$, runs with a nonzero flux $\phi$ through the sample are indicated by $\mathrm{F}\left(\phi_{0}=h c / e\right.$ is the elemental flux). NS is the number of samples (impurity configurations) considered. $A R=L_{x} / L_{y}$ is the aspect ratio of the samples and $N$ is the number of propagating modes. $E_{f}$ and $k$ are the Fermi energy and the wavevector in Anderson units $\left(E_{f}=-2-2 \cos k\right)$. $W$ is the amplitude of the on-site disorder of the run. $\tau_{W}$ is the Wigner time and $\Delta$ is the level spacing of a perfect (non-disordered) rectangle with sides $L_{x}, L_{y}$. The column showing $\frac{\tau_{W} \Delta}{\hbar} \frac{N}{\pi}$ checks the approximate validity of Eq. (9.1). $l$ is the elastic-mean-free path and $r_{c}$ is the critical angle $\eta_{c}$ (Eq. (9.10) ) scaled with the mean phase shift spacing. $\phi_{c}$ is the slope of $\sigma^{2}$ evaluated at $r_{c}$ obtained from Fig. 8. $\langle g\rangle$ is the averge dimensionless conductance and $\left\langle\delta g^{2}\right\rangle$ its variance. $|\langle\operatorname{Tr} S\rangle|$ is the average trace of the scattering matrix. 


\section{FIGURES}

FIG. 1. Typical sample geometry where the disordered scattering is confined to the region $0 \leq x \leq L_{x}$ of the wave guide. $A$ and $D$ are the amplitudes of the incoming flux, $B$ and $C$ are the outgoing amplitudes.

FIG. 2. Phase shift form factor (Eq. (5.7)) for quasi-1d conductors (upper inset) without magnetic field (R1) and with $\phi / \phi_{0}=2(\mathrm{~F} 1)$. The large- $M$ OE and UE values are given by thick solid and dashed lines. The number of impurity configurations is $N S=5000$, the aspect ratio is $A R=4$, the disorder is $W=1$ and the number of propagating modes is $N=M / 2=14$. Lower inset: histograms of the phase shift distribution normalized by the uniform value $\rho_{0}=M / 2 \pi$. The $\mathrm{R} 1$ distribution is off-set by $-1 / 3$, while the $\mathrm{F} 1$ distribution is off-set by $-2 / 3$.

FIG. 3. Phase shift form factor for quasi-1d insulators without (a) and with (b) magnetic field. Filled circles correspond to strong localization, while diamonds represent a quasi-1d metal-insulator crossover $\left(L_{x} \approx \xi\right)$ with weak disorder. Histograms in the insets show the corresponding phase shift distributions with an off-set of $-1 / 3$ for the strong disorder case and an off-set of $-2 / 3$ for weak disorder. The thick solid and dash lines in a (b) represent respectively the large- $M$ form factor of one $\mathrm{OE}(\mathrm{UE})$ and of the superposition of two independent OE (UE).

FIG. 4. Number variance, with (a) and without (b) time-reversal symmetry, as we approach the localized regime by increasing the disorder or the system length. Thick solid lines correspond

to the number variance in the orthogonal (unitary) ensembles, Eq. (6.5), while thick dashed lines correspond to the number variance of the superposition ensembles, Eq. (6.7). White symbols show the approach to the quasi-1d localized regime by increasing the length keeping the disorder weak $(W=1): A R=4$ (squares), 10 (circles) and 30 (diamonds). Filled symbols show the approach to the localized regime by increasing the disorder keeping the geometry fixed $(A R=4), W=2$ (triangles), 4 (circles), 6 (squares).

FIG. 5. Diagrams of second-order perturbation theory for the average Green function used to calculate the average of the reflection matrix elements.

FIG. 6. Average values of the diagonal reflection amplitudes as a function of the mode number for the samples scketched in the insets. The solid thick lines are guide-to-the eye with the functional form $1 / k_{a}$ and $N=14$ modes. (a) R1: $A R=4, W=1$; R8: $A R=10, W=1 ; \mathrm{R} 2: A R=4, W=1.5$. (b) R12: $A R=1, W=1, N=14$; R11: $A R=4, W=1$; R13: $A R=1, W=1.5, N=14$; R18: $A R=1, W=1.5, N=28$. For the last sample we show the average diagonal reflection amplitude for only the first half of the mode numbers. 
FIG. 7. (a) Phase shift form factors after unfolding the raw data for square samples (upper inset) for $N=14$ propagating modes. The sample with a disorder strenght of $W=1$ is represented by squares (R12) for $B=0$ and triangles (F12) for $\phi / \phi_{0}=2$. Circles represent a sample with $W=1.5$ and no magnetic field (R13). The large- $M \mathrm{OE}$ and UE values are given by solid and dashed lines. Lower inset: histograms of the phase shift distribution normalized by the uniform value $\rho_{0}=M / 2 \pi$. The distribution R12 (F12) is off-set by -0.5 (-0.75), while the distribution of R13 is off-set by -0.25 . (b) Phase shift form factors after unfolding the raw data (circles) and according to Eq. (5.7) (filled squares) for slab-shape samples (upper inset) without magnetic field (R19). Triangles: Form factors according to Eq. (5.7) for $\phi / \phi_{0}=2$ (F19). The large- $M$ OE and UE values are given by solid and dashed lines. Lower inset: histograms of the phase shift distribution normalized by the uniform value $\rho_{0}=M / 2 \pi$. The R19 distribution is off-set by -0.4 , while the F19 distribution is off-set by -0.8 .

FIG. 8. Difference between the number variance obtained in the numerical simulations and the number variance of the standard ensembles. $\sigma^{2}(r)=\Sigma^{2}(r)-\Sigma_{O E}^{2}(r)$ for samples without magnetic field and $\sigma^{2}(r)=\Sigma^{2}(r)-\Sigma_{U E}^{2}(r)$ for samples with magnetic field. Solid (dashed) lines are for samples without (with) magnetic field. Thin lines are for square geometries, R12, F12: $A R=1$, $W=1, N=14 ; \mathrm{R} 13: A R=1, W=1.5, N=14 ; \mathrm{R} 15: A R=1, W=1.5, N=20$. Thick lines are for thin slabs, R19, F19: $A R=1 / 4, W=1, N=14$.

FIG. 9. Slope of $\sigma^{2}(r)$ (obtained from Figs. 6 and 8) as a function of the inverse of the reduced critical angle $r_{c}=\left(\tau_{W} E_{c} / \hbar\right)(M / 2 \pi)$. The symbols used for the various runs are consistent with the labels of Table I of Appendix A. Samples R1-R11 are quasi-one dimensional, while samples R12-R17 represent the two-dimensional case (square geometries). The dashed straight lines are guide-to-the-eye through the two groups of data points and illustrate the approximate validity of Eq. (9.11).

FIG. 10. Energy dependence of the phase shifts for one of the quasi-1d conductors studied in Fig. 1 (aspect ratio $A R=4$, disorder $W=1$ ) in an energy range where there are $N=14$ propagating modes. 